\title{
The Lightweight Composite Structure and Mechanical Properties of the Beetle Forewing
}

\author{
Jinxiang Chen, Qing-Qing Ni and Juan Xie \\ Additional information is available at the end of the chapter
}

http://dx.doi.org/10.5772/48807

\section{Introduction}

The development of lightweight, energy-saving structures is believed to be one key solution to current world problems, such as increasing population, resource shortages, and environmental pollution. Acquiring inspiration from living creatures is an effective approach because these forms have evolved over millions of years to adapt to the natural environment [Thompson, 1945]. Examples of evolutionary adaptations provide evidence of how nature has helped organisms to overcome their structural weaknesses [Thompson, 1945; Wainwright et al., 1976; Hepburn, 1976]. Motivated by our insight that the forewings of beetles should have both high strength and minimal weight, which are needed both for defense and for flight, the first author of this paper has been studying their architecture since 1997 [Chen et al., 2000; Chen et al., 2001a; Chen et al., 2007a; Chen et al., 2007b]. However, beetle forewings were considered nonliving materials in early work [Ishii, 1982], which may be the reason why research on beetle forewings and their biomimetic applications has been limited to two-dimensional fiber orientations [Gullan \& Cranstion, 1994; Zelazny \& Neville, 1972]. To date, we have comprehensively investigated their threedimensional structures and obtained new information on their biological morphology [Chen et al., 2007b; Chen et al., 2001a; Chen et al, 2002], and we have gained new insight into their mechanical properties [Chen et al., 2000; Chen et al., 2007a; Ni et al., 2001]. Our investigations have not only confirmed the above insights but also led us to discover a new type of lightweight biomimetic composite that is more complicated and more delicate than the presently used honeycomb structure. This new composite features a completely integrated honeycomb structure with fiber-reinforced trabeculae at the corners of the honeycomb cores [Chen et al., 2005; Chen et al., 2012].

In this chapter, we present insight into the lightweight composite structure of the beetle forewing, including its mechanical properties and practical applications. 


\section{The lightweight composite structure of the beetle forewing}

\subsection{Experiment}

Two species of adult beetles, A. dichotoma and P. inclinatus, were used as experimental samples and are shown in Fig. 1(a) and (b), respectively.

Since living samples were used, their careful treatment by specialized methods to ensure that their structures would remain unchanged during the sample preparation process was highly important. Here, three specialized treatments were used for comparison of the forewing structural damage caused by removal from the living beetle body: (1) living samples (tests are conducted within 1-3 minutes); (2) traditional mistreatment; and (3) the living samples left at room temperature for one day or, in some cases, even several months. The results show that by using method (3), which may result in drying due to loss of water and partial damage of the cell membrane, the original structural integrity of the forewing still remains [Chen et al., 2002]. In this experiment, unless otherwise noted, method (3) was used for sample preparation.

The observation positions were set using Cartesian coordinates, and the names of the different regions of the forewing are shown in Fig. 1(c). The cross sections were prepared by slicing the forewing along the $\mathrm{X}$ or $\mathrm{Y}$ line, after which the inner structure of the forewing was observed by removing a portion of the lower lamination with forceps.
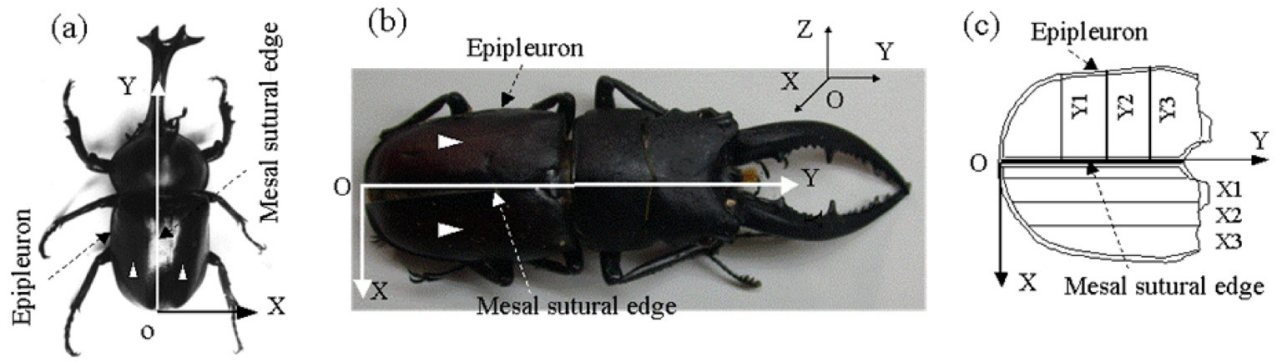

Figure 1. Two species of adult beetle and the Cartesian coordinates ( $\triangle$, the fore-wings). (a) male $A$. dichotoma, (b) male P. inclinatus, (c) observation sites.

A strong alkali can be used to examine the composition of the forewing; the chitin fibers do not readily dissolve in the alkaline solution, while the proteins dissolve easily. Once the protein matter has been removed by the alkali solution, the structures of the trabeculae can be readily observed. Therefore, some samples were prepared by boiling in a $10 \% \mathrm{KOH}$ solution [Zelazny \& Neville, 1972] for three hours. This treatment does not affect the orientation of the chitin fibers.

The main experimental instruments used in this study were an environmental scanning electron microscope (Nikon ESEM-2700 at $20 \mathrm{kV}$ and $460 \mathrm{~Pa}$ ) and a general SEM (Hitachi, S510 at $15 \mathrm{kV})$. 


\subsection{Results and discussion}

\subsubsection{General structure of the beetle forewing}

The forewing photographs of $A$. dichotoma are presented in Fig. 2(a-e). Fig. 2 (a, c) and Fig. 2 $(b, d, e)$ contain images captured with a camera under a penetrating light with a short focus
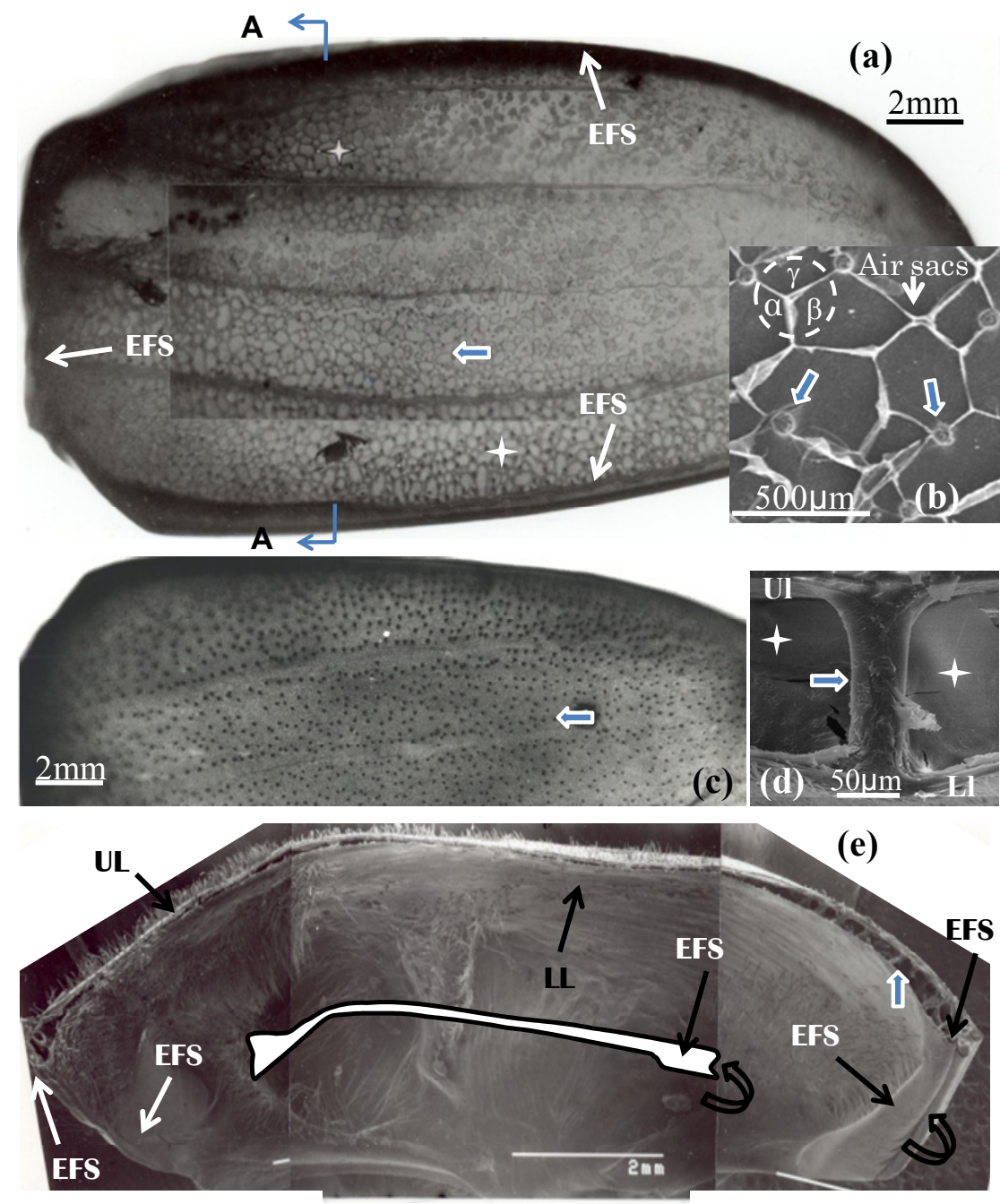

Figure 2. The integrated structure and edge sealing of the forewing of A. dichotoma. (a) Meshwork and trabeculae (dots) in a fresh forewing, (b) the internal structure and the lower lamination were eliminated by forceps, (c) distribution of trabeculae (dots) in the forewing (following $10 \% \mathrm{KOH}$ treatment), (d) a trabecula, (e) the cross sectional view of the A-A section, and the middle part of the figure is a simple sketch of the cross section. EFS: edge frame structure, asterisk: air sacs, thick arrows: trabeculae, round arrows: concavo-convex junction, Ul: upper lamination, Ll: lower lamination. 
time and with a SEM, respectively. The many lines Fig. 2(a) apparent in those photos are actually air sacs or tracheae [Chen et al., 2002]. The images in Fig. 2 show the samples after three hours of boiling in the $10 \% \mathrm{KOH}$ solution. The air sacs and the tracheae that can be observed in Fig. 2(a) have disappeared here because they were dissolved during the $\mathrm{KOH}$ treatment. However, many black solid dots (Fig. 2c) are visible, each of which represents an individual trabecula [Chen et al., 2002].

As shown in Fig. 2 (e), the entire beetle forewing is composed of a single type of frame structure. Fig. 2 (e) shows an electron micrograph of this frame structure at an X-Z plane cross section (see Fig. 1). It was found that the forewing cross section consists of three subdivisions; the upper lamination, the lower lamination and the central void, with the large voids located at both the mesal sutural and the epipleuron edges (see Fig. 1). The middle portion of the forewing is a sandwich structure containing a wide void area.

\subsubsection{Trabecular structure in the beetle forewing}

The longitudinal shapes of typical forewing trabeculae in A. dichotoma are shown in Fig. 3 (a, b), and a cross section is shown in Fig. 3(c). The trabecular size and shape can change according to location in the forewing. Both trabeculae with a straight shape (Fig. 3a) and with a curved shape (Fig. $3 b$ ) were observed, although the majority of the trabeculae were of the straight shape kind. The cross section of any trabecula is similar to the circular structure presented in Fig. 3(c). The diameter of a trabecula is larger near the locations of joints with the upper and lower laminations and is smaller in the void region between.
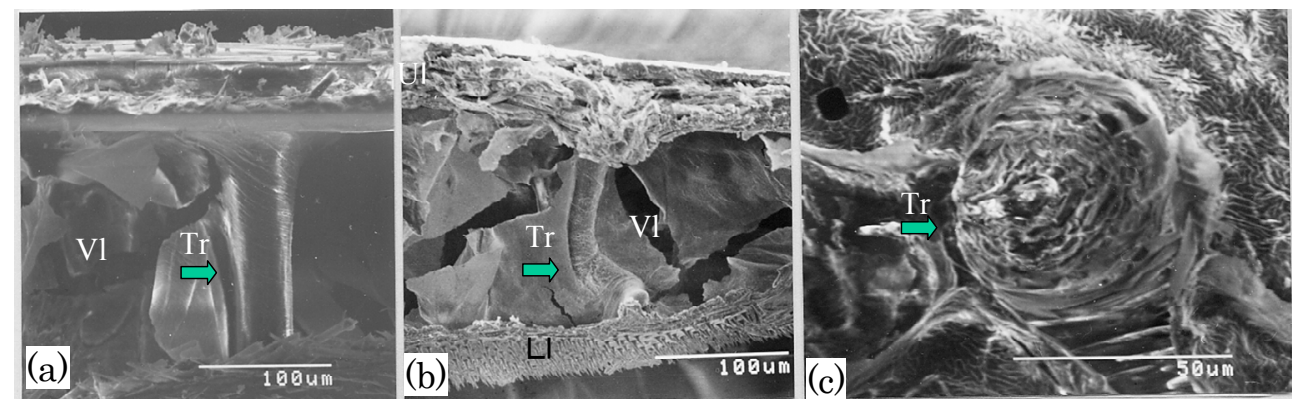

Figure 3. The trabecular ( $\mathrm{Tr}$ ) shapes. (a) Straight cylinder, (b) curve cylinder, (c) section view. Ul, upper lamination, Vl, void lamination, Ll: lower lamination.

Fig. 4 shows longitudinal sections of the trabeculae, which allow for clear observation of the chitin fiber arrangement within the trabeculae. In Fig. 4(a), the chitin fibers Fb (or marked $t 5$ ) form a distinct layer within the upper lamination. It should be noted that the fiber angle within this layer is nearly perpendicular (at 90 degrees) to the paper and that the neighboring chitin fiber layers ( $\mathrm{Fl}$ or $\searrow$ ) are instead horizontally arranged and connected with the trabecula in a curving manner. This connection between the chitin fiber layers in the upper lamination and the trabecula can also be seen clearly in Fig. 4(b). Fig. 5 shows micrographs of trabeculae after having been boiled in the $10 \% \mathrm{KOH}$ solution for three hours. 
Due to removal of the protein matter, the chitin fiber arrangement on the outside of the trabeculae can easily be observed. The chitin fibers in the left image (Fig. 5a) are in a straight arrangement, while the fibers in the right image (Fig. 5b) have a spiral arrangement on the trabecular surface. The central regions of the trabeculae become hollow (Fig. 5c) due to dissolution of the internal proteins during the $\mathrm{KOH}$ solution treatment. Thus, the central portion of the trabecular shaft is composed mainly of protein [Chen et al., 2001b].
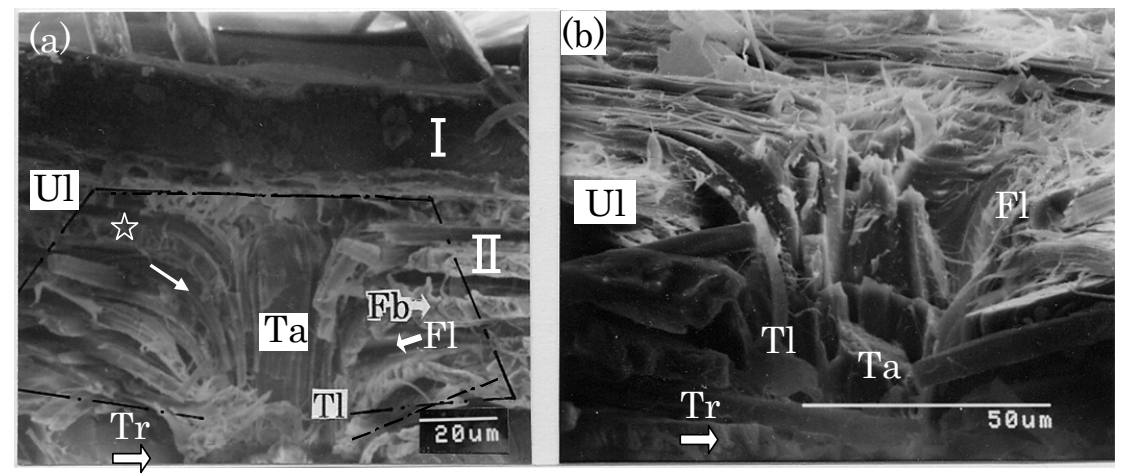

Figure 4. The trabecular structure in the forewing of A. dichotoma. (a) longitudinal section with the trabecular root marked by the lines; (b) a naturally rupture trabecula. Ul, upper lamination. Fl, fibers from left to right. Fb, fibers from front to back. Ta, trabecular central part. Tl, trabecular cylindrical layers. Tr, trabecula.
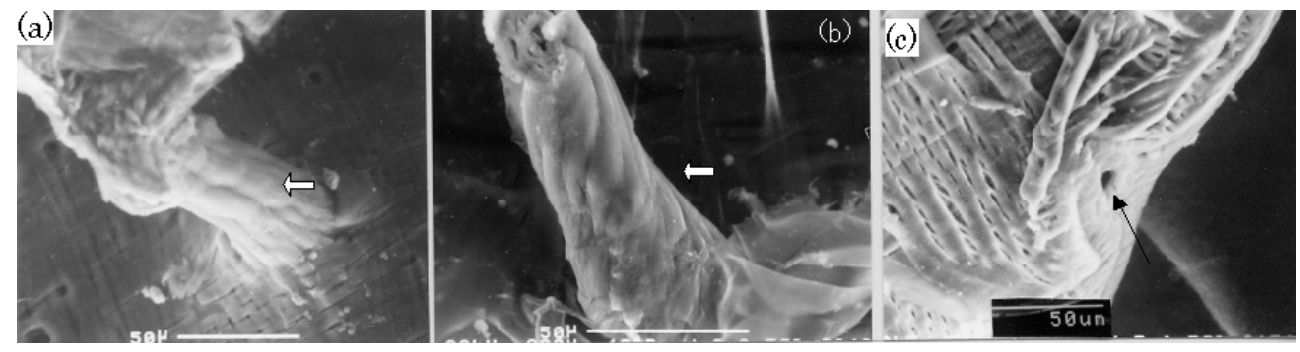

Figure 5. Trabecular structures treated with $\mathrm{KOH},(\mathrm{a})$ the straight orientation (b) the spiral curved orientation of the chitin fiber at the outside, $(c)$ the hollow $(\nwarrow)$ at the center of the trabecular root.

\subsubsection{Schematic model of the forewing structure}

Based on these observations and analyses of the beetle forewing and its inner trabecular structures, it is evident that the general forewing structure in A. dichotoma is a sandwich frame structure with a central void region. However, the details of the forewing microstructure are extremely complex. These models for the forewing and trabecula structures are proposed to assist in the development of biomimetic composite structures. According to our observations, we have developed a sandwich plate structure model with a central void layer and trabecular struts [Chen et al., 2005]. It is well known that sandwich plates with a central void layer are typical of lightweight structures and already have been applied in many fields [Jung \& Aref, 
2005; Matsunaga, 2002; Nguyen et al., 2005; Mania, 2005; Hosur et al., 2005]. The presence of the sandwich plate structures with trabeculae in the beetle forewings indicate that the beetle wings have been evolutionarily optimized for competition in nature.

Fig. 6 shows the sandwich plate model that was inspired by the forewings of the beetles. The plate consists of upper and lower lamination layers and a core layer with trabeculae and honeycomb walls (Fig. 6a). There are many trabeculae positioned throughout the honeycomb, and they are located at the corners of the honeycomb units (Fig. 6b).
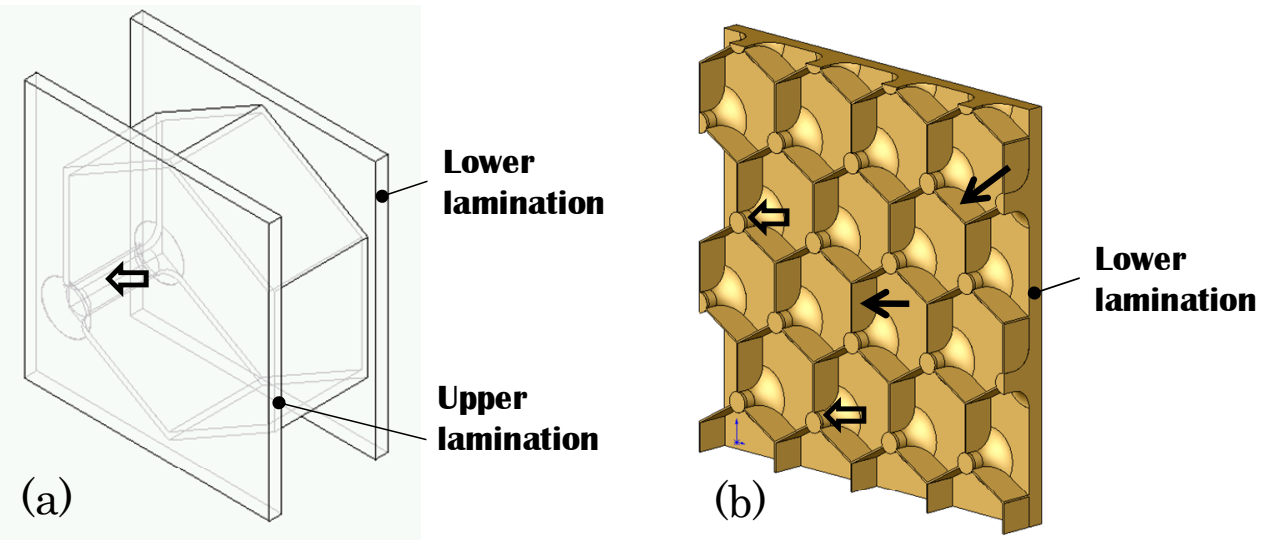

Figure 6. Three-dimensional sketch of a trabecula-honeycomb cell. (a) A cell. (b) the assembly of cells without an upper lamination layer. The thin and thick arrows indicate the honeycomb wall and the trabeculae, respectively.

\subsubsection{Schematic model of the trabecular structure}

Based on our observations, a schematic structural model of the trabeculae in A. dichotoma was generated and is shown in Fig. 7. This model indicates the following: (1) the substance in the central part of the trabecula is protein (see thin arrow in Fig. 7a); (2) The upper and lower laminations are comprised of chitin fiber layers that either extend into the trabeculae continuously in a curved shape (see Fl in Fig. 4a and Fig. 7) or connect to the trabeculae by surrounding the circumference of the trabecular shaft in a spiral fashion (see $\mathrm{Fb}$ in Fig. $4 \mathrm{a}$ and Fig. 7). Through peeling tests, we have confirmed that, when comparing the trabeculae of the upper and lower laminations, the peeling resistance of trabeculae in a general region is approximately three times greater and in a local region is approximately thirty times greater [Ni et al., 2001]. Thus, the use of the trabecular structures to join the upper and lower laminations effectively increases the interlaminar strength of the composite structures.

\subsection{Conclusions}

To develop lightweight biomimetic composite structures, the forewing structures of $A$. dichotomais were investigated. Structural models for both the beetle forewings and the forewing trabeculae were also established, and the results obtained are as follows: 

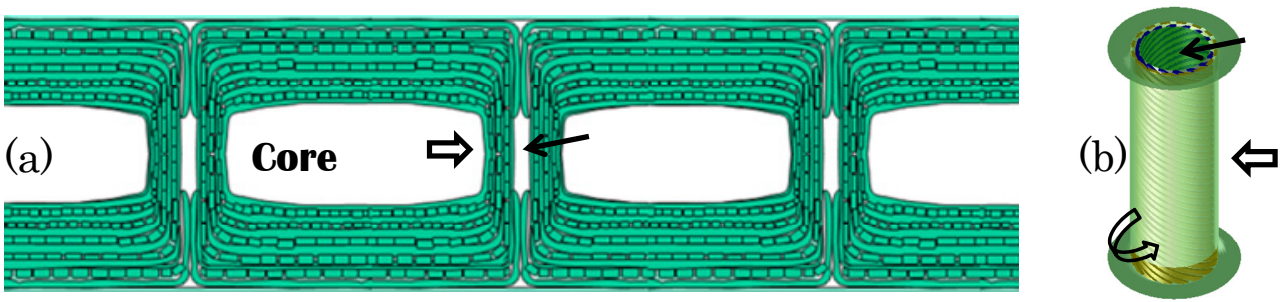

Figure 7. Schematic view of reinforcing fibers. a, Cross section of a forewing. $b$, Three-dimensional view of a trabecula. The thin and thick straight arrows show a void (protein) inside a trabecula and itself, respectively. The circular arrow shows a spiral fiber surrounding the trabecula (the lower part shows the structure of a trabecula without a protein).

1. It was found that both species of beetle forewings have a sandwich plate structure and a frame structure with a central void layer and interconnecting trabeculae. These characteristics are typical of lightweight composite structures.

2. The chitin fibers in the trabeculae connect with a curved shape continuously to the chitin fibers on the upper and lower laminations, and it is clear that this structure greatly increases the peeling resistance of the laminated composite structures.

\section{Interlaminar reinforcement mechanism of the beetle forewing}

The fracture toughness of fiber-reinforced composite materials used in engineering applications mainly depends on the interlaminar and/or intralaminar fracture toughness values, with the interlaminar and/or intralaminar interface tending to be the critical location of weakness. Investigations of the interfacial behavior have been undertaken by many researchers, such as evaluation of the interlaminar and/or intralaminar fracture toughness [Iwamoto et al., 1999a], the interlaminar delamination resistance [Turss et al. 1997], and the role of bridging fibers in crack propagation [Sekine \& Kamiya, 1987; He \& Cox, 1998]. In recent years, 3D textile-reinforced composites have been developed toward improving the interlaminar and/or intralaminar fracture toughness values. On the other hand, many examples of composite structures already exist in nature, and their structures have already been optimized by evolutionary pressures over the course of a deep ecosystem history.

This section focuses on the mechanical properties and the three-dimensional interlaminar reinforcement mechanism of the A. dichotoma beetle forewing.

\subsection{Experiment}

The specimen utilized was the living forewing of the adult male A. dichotoma beetle (Fig. 1a, $\Delta$ arrowhead). The width of the fresh specimen was $2.1 \mathrm{~mm}$. A tensile device (SHIMAZDU, AUTOGRAPH DSC-10T) with a custom low load cell was used for the interlaminar peeling test, which was conducted with zipper forceps, as developed by the authors. The tensile loading direction was vertical to the peeling surface, as shown in Fig. 8(a). To prevent sliding of the specimen at the zippers, the inner surface of the forceps was adhered to the specimen 
with glue tape. The peeling schematic is indicated in Fig. 8(b). In the present study, 10 specimens were tested at a tensile speed of $5 \mathrm{~mm} / \mathrm{min}$ and a peeling distance of $7 \mathrm{~mm}$.

(a)

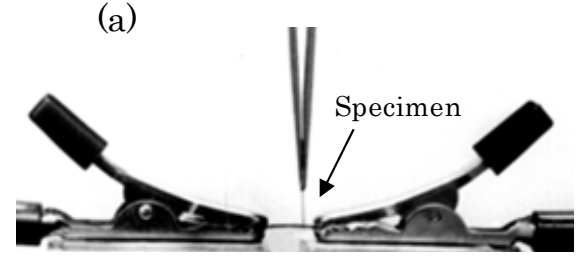

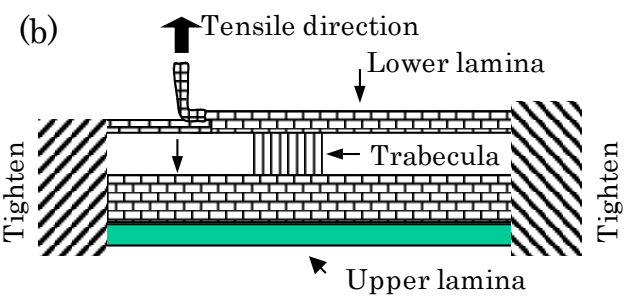

Figure 8. A peeling experiment; (a) peeling device, (b) a peeling schematic.

\subsection{Results and discussion}

\subsubsection{Peeling characteristics of the forewing of A. dichotoma}

Micro Fractograph of Peeling Surface Fig. 9 presents a micrograph of a forewing after the peeling test by illuminating the specimen from behind with penetrating light, which is a new technique developed for observation of the forewing structure. Many black points and ducts can be observed in this image, where the black points can be interpreted as trabeculae and the ducts, as tracheas. Fig. 10 (a) is a micrograph taken at the $\Delta$ arrowhead mark in Fig. 9 and shows the fracture characteristics of the first trabecula during the peeling process. The peeling test was started by applying pulling force to the lower lamina of the forewing. When the delamination crack tip met the first trabecula (Fig. 10a, $\Delta$ arrowhead), a portion of the lower lamina tore, while the remaining portion was held in tension. In this manner, the peeling tip continued to develop and propagate between the chitin fiber laminae. A fracture micrograph illustrates a peeling surface in the region of the $\boldsymbol{\Delta}$ arrowhead marked in Fig. 9 and has been shown in Fig. 10(b). The resulting fracture patterns on the peeling surface had an appearance similar to that of a tree root (Fig. 10b, $\Delta$ arrowheads) with details of this phenomenon presented in Fig. 11(b) and Fig. 13.

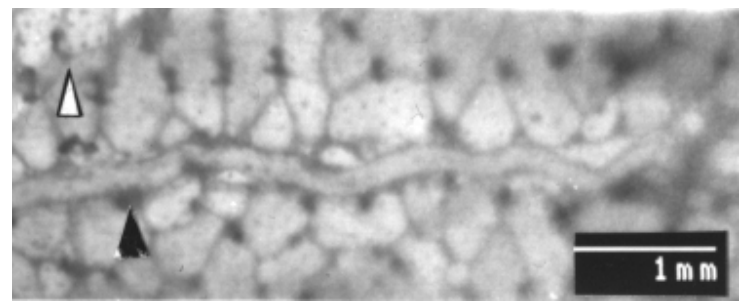

Figure 9. A photograph of a fore-wing after peeling test and black points are trabeculae.

Fig. 11(a) illustrates the fracture pattern of the peeling surface without any trabeculae present. Although the chitin fibers are arranged in rows ( $\Delta$ arrowhead), some of have torn ( $\Delta$ arrowhead) during the peeling process. Fig. 11(b) shows a fracture micrograph of the chitin fibers at a trabecular root. It was that the chitin fibers ( $\Delta$ arrowhead) formed very thin 

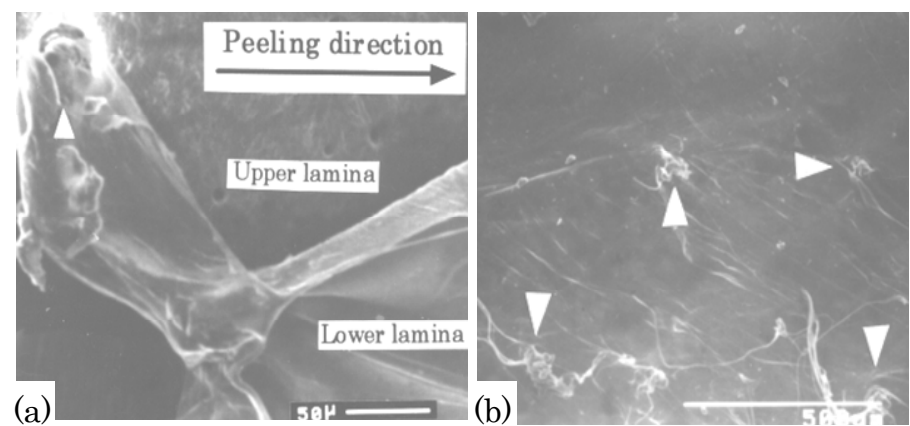

Figure 10. Fractographs of a forewing; (a) fracture of first trabecula, $\Delta$ : trabecula, (b) a peeled chitin fiber lamina, $\Delta$ : roots of the trabeculae.

layers here, with Fig. 11(c) illustrating a side view of the peeling process. When the peeling tip had propagated about half of the thickness of the lower lamina, a few bridging fibers can be seen between the lower and upper peeling surfaces.

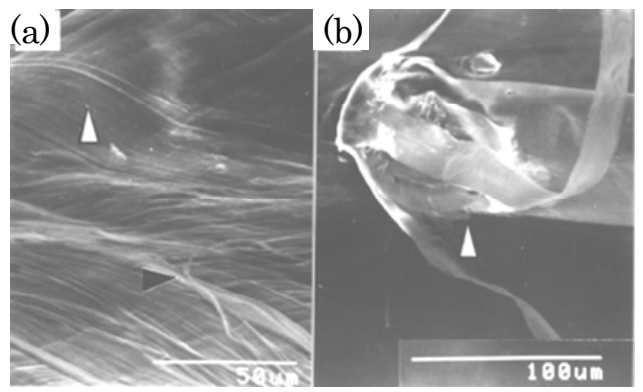

(c)

Figure 11. Fractographs of a forewing; (a) peeled surface without a trabecula, (b) trabecula root and thin chitin fiber layers, (c) a peeling process from a side view. $\Delta$ : bridging fibers.

\subsubsection{Load peaks and trabeculae in a peeling test}

A representative load-displacement curve, with load $F$ and displacement $\delta$, is presented for a forewing peeling test in Fig. 12(a). The force, $F(\delta)$, was proportional to the resistance force because the tensile direction was held vertical to the peeling surface, as described above. Here, the $F-\delta$ curve is named the "peeling test" and the curve is given by $F(\delta)$. Many individual load peaks were present in the peeling curve, and their shapes were similar to the standard load peak shape, although their peak values were quite different. In the peeling test, the minimum value of each ravine was not equal to zero but instead held a nearly constant value during the peeling process. Here, the $\mathrm{j}^{\text {th }}$ values of the load peak and the ravine minimum are indicated by $F_{\mathrm{j} 1}(\delta)$ and $F_{\mathrm{j} 0}(\delta)$, respectively.

The number of individual load peaks in the $F-\delta$ peeling test was compared to the number of trabecular roots, which were observed by SEM, and these values were found to be nearly equal [Ni et al., 2001]. Accordingly, it was determined that each trabecula contributed a 
single load peak in the peeling curve. In other words, the load peaks resulted from breakage of the individual trabeculae during the peeling process.
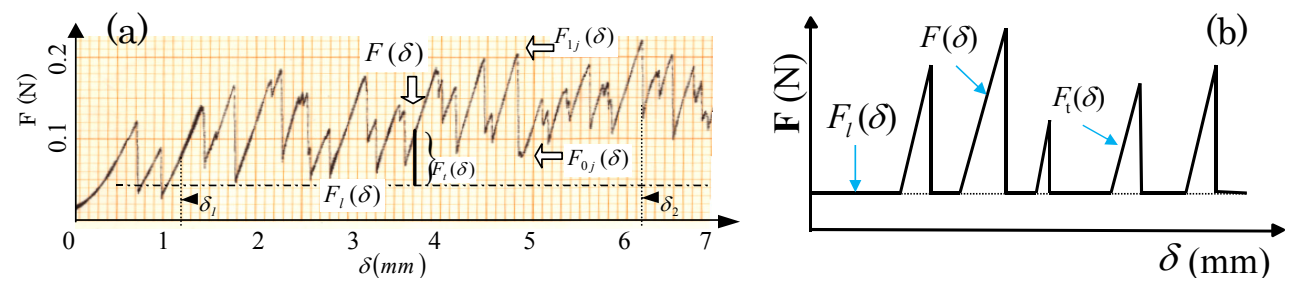

Figure 12. A representative load-displacement curve (a) and its model (b) in a forewing peeling test.

Peeling Curve As mentioned above, the load peaks in the $F-\delta$ peeling curve were attributed to specific contributions from the individual trabeculae. Based on this fact, the peeling curve, $F(\delta)$, was considered to be comprised of two components, i.e., $F_{l}(\delta)$ (Fig. 12a) due to interlaminar delamination of the chitin fiber laminae and $F_{t}(\delta)$ due to trabecula failure. Thus, $F(\delta)$ may be represented by equation (1):

$$
F(\delta)=F_{l}(\delta)+F_{t}(\delta)
$$

$F_{l}(\delta)$ depends mainly on the properties of the chitin fibers, the protein matter and fiber adhesion. If the interlaminar debonding force, $F_{l}(\delta)$, was constant during the peeling process and the trabeculae were distributed along only one line in the propagation direction, then a schematic of the peeling curve can be represented as Fig. 12(b). In other words, each load peak, $F_{t}(\delta)$, results from the fracture of one trabecula, and the line, $F_{l}(\delta)$, at the ravine minimum results from the delamination resistance between chitin fiber layers without the trabeculae.

However, the horizontal line, $F_{l}(\delta)$, cannot be observed in the $F-\delta$ peeling curve as indicated in Fig. 12. This is attributed to the trabeculae being distributed intermittently throughout the plane (Fig. 11c) with the bottom of a ravine, $F_{\mathrm{j} 0}(\delta)$, generated from the delamination of laminar fibers that are piled upon each other. In comparing Fig. 12 (b) with Fig. 12 (b), it was not possible to fully distinguish $F_{l}(\delta)$ and $F_{t}(\delta)$. However, the value of $F_{l}(\delta)$ should be less than or close to the minimum of all ravine bottoms, $F_{\min 0}$, i.e.,

$$
F_{l}(\delta) \leq F_{\min 0}=\operatorname{Min}\left\{F_{\mathrm{j} 0}(\delta)\right\}
$$

where $F_{\min 0}$ is the maximum of $F_{l}(\delta)$ in the following calculation.

\subsubsection{D Reinforcement mechanism of the beetle forewing}

Fracture Type of Trabecular Roots Three representative fracture modes of the trabecular roots were observed during the forewing peeling test (Fig. 13). Fig. 13(a) presents type A, which possesses a convex shape, and also shows a schematic of the longitudinal section shown in the fractograph. In type $\mathrm{A}$, trabecular roots in the lower laminae remained 
following peeling such that the setae ( $\boldsymbol{\Delta}$ arrowhead) could be observed. In type C, a smooth surface (Fig. 13c) has had the trabecular roots in the lower laminae completely removed. In contrast to this, type B was between type A and type C (Fig. 13b). Although three types of failure shape for trabecular roots were observed, the load peaks in the peeling curve were similar to each other. In the following section, possible reasons for this are considered.
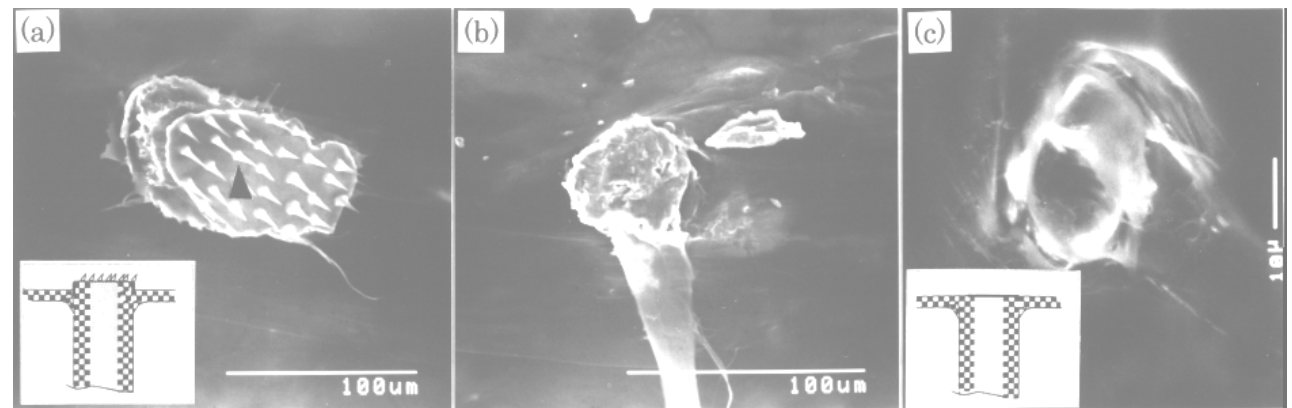

Figure 13. Fractographs of three typical trabecular root.

According to the trabecular structure, the chitin fibers within the trabeculae are thin, continuous, and curved (Fig. 14, $\mathbf{\Delta}$ arrowhead) and interconnected with each other between the chitin fiber laminae. During the peeling process, the peeling tip continues to propagate horizontally (see Fig. 11c) in the absence of a trabecula. When the peeling tip encounters a trabecula, however, its progress is impeded by the chitin fibers of the trabecular; meanwhile, the peeling tip around the outside of the trabecular root continues to propagate, which results in a stress concentration at the trabecular root itself. That is, the chitin fibers of the trabecula initially withstand the higher stress and then become stretched into a straight line until the trabecula root fails (Fig. 14a). Therefore, the chitin fibers within the trabecular root

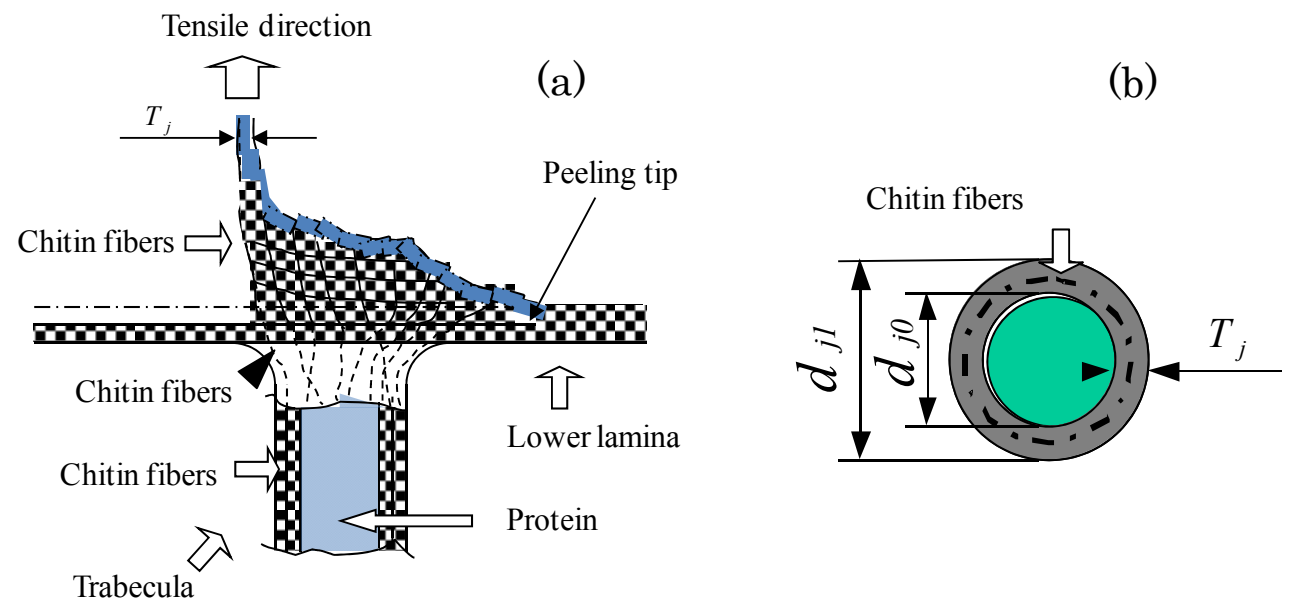

Figure 14. A chitin fiber reinforcement model (a) and a cross sectional trabecular model (b). 
fracture in tension. Thus, the chitin fibers in the trabeculae have a high resistance to the applied load, and their failure results in the load peaks noted in the $F-\delta$ peeling curves as shown in Fig. 12. This also results in similar peeling curve peak shapes for the three fracture types (Fig. 13).

Failure Strength of Chitin Fibers in the trabecular root The failure strength of chitin fibers in the trabecular root has been discussed using the proposed models (Fig. 14). Using equation (1), the load peaks, $F_{j 1}(j=1,2, \ldots, \mathrm{n}$, the number of trabeculae), and the resistance force, $F_{l}(\delta)$, of the interlaminar delamination in the peeling curve, the average failure strength, $\sigma_{b}$, of the chitin fibers in the trabeculae was calculated as follows:

$$
\overline{\sigma_{b}}=\frac{\sum_{j=1}^{\mathrm{n}}\left[F_{\mathrm{j} 1}-F_{l}(\delta)\right] / n}{\sum_{j=1}^{\mathrm{n}} S_{j} / n}
$$

where $S_{j}$ is the failure area of the chitin fibers and was calculated using the thin ring area of chitin fiber layers within the trabecular root (Fig. 14b). The thickness of the thin ring was considered to be equal to the chitin fiber lamina thickness, $T_{j}$, and $S_{j}$ was given by equation (4)

$$
S_{j}=T_{j}\left(d_{j 1}-T_{j}\right) \pi
$$

The justification for using the cross section of the thin ring area is given as follows: first, the trabecula consists of two sections, which include the central part that is mainly protein and the outside that is chitin fiber laminae. This result was confirmed by treating the forewing with $10 \% \mathrm{KOH}$ at $100^{\circ} \mathrm{C}$ for 3 hours [Iwamoto et al., 1999b]. After treatment, an empty space was observed in the central region of the trabecula (see Fig. 5c, arrowhead part), which was attributed to dissolution of the protein in the $\mathrm{KOH}$ solution. Therefore, it is clear that the central region of each trabecula contains no chitin fibers and is instead mainly composed of protein. Second, considering the fracture of chitin fibers during the peeling process (see Fig. 14a), when the peeling tip of the chitin fibers progresses around the outside of the trabecular root, the chitin fibers at the remaining intact side bear a higher applied load than those in the delaminated side. However, in the cross section of the trabecular root thin ring, the stress is uniformly accommodated; in particular, this is true after the outside chitin fiber laminae of the trabecula are peeled apart (delaminated) because the chitin fiber layer is very thin. This hypothesis was supported by the fact that the load peaks in the peeling curve dropped when a trabecular root fractured during the peeling process (Fig. 12). Third, the trabecular diameter in the forewing of $A$. dichotoma is very small and only on the order of tens of microns. In light of the above reasons, and for simplicity, equation (4) was thus adopted.

Moreover, for equation (3), only data collected in the region between $1 \mathrm{~mm}$ and $6 \mathrm{~mm}$ of displacement were used because the peeling front had not always progressed through the full specimen width in the initial and final failure regions. Thirty load peaks in the region of 
$1 \mathrm{~mm}$ to $6 \mathrm{~mm}$ were used for the calculation, although there were 44 load peaks in the total peeling curve as shown in Fig. 12. As a result, the trabecular root failure load and the resistance force, $F_{l}(\delta)$, of interlaminar delamination were found to be $0.156 \pm 0.029 \mathrm{~N}$ and $0.049 \mathrm{~N}$, respectively. The thickness of the peeled chitin fiber laminae, $T_{j}$, was $4.5 \mu \mathrm{m}$, which remained nearly constant in the entire peeling region from $1 \mathrm{~mm}$ to $6 \mathrm{~mm}$.

Next, the external diameter of the peeled trabecular root, $d_{j 1}$, was determined. In practice, the cross section of the trabecular root is not circular but instead is closer in shape to an ellipse. Thus, the average of the long and short axes of the ellipse was used as $d_{j 1}$. The results of $d_{j 1}$ as measured by SEM indicated the average external diameter of 30 trabecular roots was $55 \mu \mathrm{m} \cdot$ Using all of the results obtained above, the failure strength, $\overline{\sigma_{b}}$, of the chitin fibers within the trabecular root was calculated as approximately $149 \mathrm{MPa}$. This result is close to the tensile strength of $147 \mathrm{MPa}$ in the same $0^{\circ}$ direction of the chitin fibers. Therefore, during the peeling test, the failure strength of the chitin fibers within the trabecular root ultimately resulted from tensile fracture of the chitin fibers, as mentioned above.

Interlaminar Reinforcement Mechanism The chitin fibers of the forewing trabecular root were found to have the following characteristics: a curved and continuous shape; bonded to both chitin fiber laminae and to each other; with the angle of about $90^{\circ}$ between the lower laminae and the trabecula. The increment of interlaminar strength is due to the tensile failure of the chitin fibers within the trabecular root, as determined by the peeling process. These results indicate a creative approach for researchers to overcome the interfacial weakness issues currently associated with laminated composite structures.

Strengthening Effects due to the Trabeculae The strengthening effects due to the forewing trabeculae were investigated. Here, the local strengthening effect, $\lambda_{l}$, is calculated by equation (5), i.e., $\lambda_{l}$ is the ratio of the average failure load of the chitin fibers in the trabecular root due to the resistance force of the interlaminar lamination, $F_{l}(\delta)$, in units of inverse length.

$$
\lambda_{l}=\overline{\sigma_{b}} \cdot T_{j} / \frac{F_{l}(\delta)}{B} \cong 29
$$

Using the data presented in Fig. 12, the average failure load of the chitin fibers in the trabecular root, $F_{t}(\delta)$, the resistance force of the interlaminar lamination, $F_{l}(\delta)$, and the specimen width of $B=2.1 \mathrm{~mm}$ were all calculated. The local strengthening effect, $\lambda_{l}$, was found to have a value of approximately 30. Therefore, the 3D strengthening effect of the interlaminar structure is very pronounced, specifically due to the curved and continuously shaped chitin fibers, which are shown in Fig. 14.

The strengthening effect for the whole specimen that is due to the trabeculae can be evaluated from instances where the trabeculae are or are not present to provide resistance during the peeling process. The whole strengthening effect, $\lambda_{m}$, was calculated using equations (6) and (7): 


$$
\begin{gathered}
\lambda(\delta)=\frac{F(\delta)}{F_{l}(\delta)} \geq \frac{F(\delta)}{F_{\min 0}} \\
\lambda_{m} \geq \frac{\int_{\delta_{1}}^{\delta_{2}} F(\delta) \mathrm{d} \delta}{\int_{\delta_{1}}^{\delta_{2}} F_{\min 0} \mathrm{~d} \delta}=\frac{\int_{\delta_{1}}^{\delta_{2}} F(\delta) \mathrm{d} \delta}{F_{\min 0} \cdot\left(\delta_{2}-\delta_{1}\right)}
\end{gathered}
$$

where $\lambda(\delta)$ is the strengthening effect at a peeling distance, $\delta$. In this paper, the average of 10 specimens, $\overline{F(\delta)}, \overline{F_{\min 0}}, \bar{\lambda}_{m}$ and the standard deviations for $F(\delta), F_{\min 0}(\delta), \lambda_{m}$ were calculated. The value of $\overline{F(\delta)}$ is given by equation (8)

$$
\overline{F(\delta)}=\sum_{i=1}^{10}\left[\int_{\delta_{1}}^{\delta_{2}} F_{i}(\delta) \mathrm{d} \delta /\left(\delta_{2}-\delta_{1}\right)\right] / 10
$$

The entire strengthening effect of the interlaminar strength due to the trabeculae in the chitin fiber laminae was approximately 3 times as large as that in the chitin fiber laminae without the trabeculae. Therefore, the trabecular structure of the forewing is not only lightweight but also high-strength.

\begin{tabular}{|c|c|c|c|}
\hline Object & $\overline{F(\delta)}(\mathrm{N})$ & $\overline{F_{\min 0}}(\mathrm{~N})$ & $\overline{\lambda_{m}}$ \\
\hline Average & 0.148 & 0.051 & 3.2 \\
\hline Standard deviation & 0.047 & 0.019 & 1.1 \\
\hline
\end{tabular}

Table 1. Strength ratio and its standard deviation by trabecula

\subsection{Conclusions}

A peeling test between the chitin fiber laminae of the A. dichotoma forewing was conducted, and the reinforcement mechanism, especially for the 3D interlaminar reinforcement, was investigated with the following results.

1. Many load peaks were observed in the peeling curves with their number being equal to the number of trabeculae found in the chitin fiber laminae. This finding demonstrated that the fracture of each trabecula contributed to a single load peak in the peeling curve, which ultimately contributed to the interlaminar strength. Furthermore, the characteristics of the peeling curve depended on the density and 2D distribution of the trabeculae within the forewing.

2. The chitin fibers were interconnected between the chitin fiber laminae and the trabeculae, and they were curved and continuously shaped where the trabeculae were bonded to the chitin fiber laminae. The fibers were distributed in two dimensions within the plane of each chitin fiber lamina. The chitin fibers broke in tension during the peeling process and exhibited a high resistance to the applied load.

3. The amount of the interlaminar strength that was due to the trabeculae in the chitin fiber laminae was approximately 3 times greater within the whole region and about 30 
times greater in the local region compared to that in the chitin fiber laminae without any trabeculae. Based on the above results, this strong natural reinforcement mechanism was understood, and a model for the reinforcement mechanism was proposed.

\section{Optimal composite structures in the forewings of beetles}

Within the forewings of insects, both the laminated arrangement of the chitin fibers [Gullan \& Cranstion, 1994; Leopoldj et al., 1992; Banerjee, 1988; Zelazny \& Neville, 1972] and the mechanical characteristics of these equiangular laminating layers of the biomimetic composites [Tanimoto et al., 1998; Ben et al., 1998; Masuda et al., 1995] have been reported on previously. The authors have reported the presence of non-equiangular laminating structures in the forewing of A. dichotoma [Chen et al., 2001a]. It is well known that the somatotypes of beetles differ from one another due to their sex, with the larger male bearing a horn. In the present study, based upon previous work on the characteristics of somatotype beetles, the forewing, the tensile fracture force of the forewing and its suitability for biomimetic studies have been examined for male and female beetles. Both optimal structures of the fiber's cross section and the typical reticular cross-linking of twodimensional reinforced structures within the beetle's forewing are discussed.

\subsection{Experimental specimens and methods}

Two types of forewing specimens were selected from both male and female A. dichotoma, as shown in Fig. 15(a) and Fig. 1(a). The experimental bio-samples included a total of 12 males and 12 females with measurements of each beetle's weight, its forewing dimensions and its physical size.
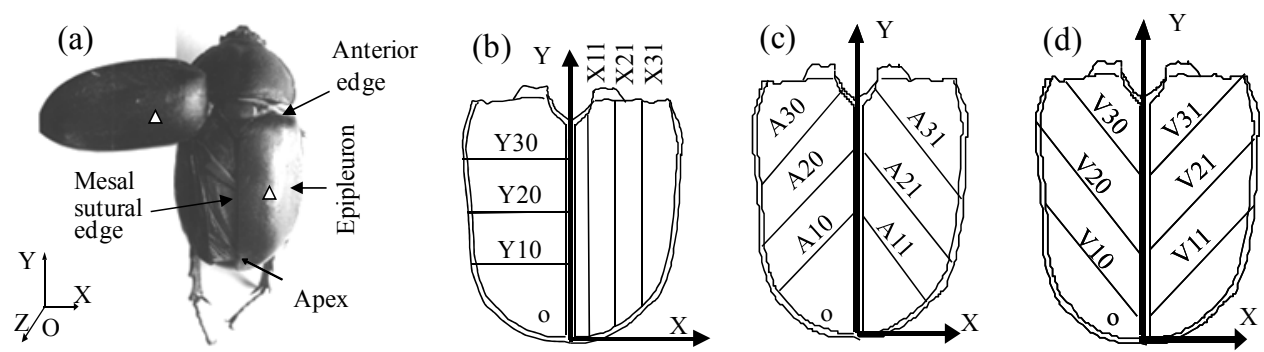

Figure 15. Female beetle (a) and example position in (b) $0^{\circ}$ or $90^{\circ}$, (c) $45^{\circ}$, (d) $135^{\circ}$ of the beetle forewing.

The different orientations of the tensile test specimens were prepared as shown in Fig. 15. The tensile test specimens measuring $4 \mathrm{~mm}$ wide were then sliced and prepared by a special parallel cutting blade [Chen et al., 2001b]. A total of 22 test specimens for both the left and right forewings were prepared. Under the conditions of constant room temperature and humidity $\left(20 \pm 2^{\circ} \mathrm{C}, 60 \% \pm 5 \% \mathrm{RH}\right)$, the tensile test was carried out on a Shimazu AUTOGRAPH DSC-10T with a special low load cell and jig at a tensile strain rate of $0.5 \mathrm{~mm} / \mathrm{min}$, where the distance between clamps was $5 \mathrm{~mm}$. Additionally, a special clamp head was attached with 
cloth tape to prevent failure. After the tensile test, the thickness of both the upper and lower lamination layers were measured at four points using the ESEM-2700, and the tensile strength of the specimens were evaluated by taking into account the average thickness of each specimen.

\subsection{Experimental results and discussions}

\subsubsection{Somatotypes}

Table 2 shows the measurement results of the somatotypes of A. dichotoma, along with the weight and size of the forewing. This also includes the mean values and the standard deviation values (STDEV).

To identify the sex-related differences between the male and female A. dichotoma, a T-test [Yoshikawa et al., 1985] was performed on these experimental results, comparing sample group A (male) to sample group B (female). The results of the T-test are shown in Table 2. When the probability value is $\mathrm{P}\left(\mathrm{t}_{0}\right) \leqq \alpha$ (generally $\alpha=0.05$ at normal significance level), there exists a significant difference and $\mathrm{a}{ }^{*}$ is labeled in that specific cell of Table 2 . It is clear that both the weight and physical size of the male A. dichotoma are larger than the weight and size of the female. The male forewing measures $28.7 \mathrm{~mm}$ in length and $14.8 \mathrm{~mm}$ in width, whereas the female forewing measures only $28.3 \mathrm{~mm}$ in length and $13.6 \mathrm{~mm}$ in width. The significant differences indicate that the male is larger than the female; however, the forewings of the male and female beetle have a similar weight. In fact, females have a forewing weight of $0.160 \mathrm{~g}$ and males have a forewing weight of $0.151 \mathrm{~g}$; surprisingly, the female beetles weigh slightly more than the males, according to this experimental data.

Compared to female $A$. dichotoma, males have a larger somatotype and physical size. However, the male also has a lighter forewing. It is thus supposed that there exist certain physical differences between males and females in A. dichotoma forewings in features such as thickness, laminated arrangement and laminated layers. Accordingly, these differences between male and female beetles should be considered when their forewing structures and mechanical properties are investigated.

\begin{tabular}{|c|c|c|c|c|c|c|}
\hline \multirow{2}{*}{$\begin{array}{c}\text { Beetle } \\
\text { AVERAGE } \\
\text { STDEV }\end{array}$} & \multicolumn{2}{|c|}{ Weight $(\mathrm{g})$} & \multicolumn{2}{c|}{ Length $(\mathrm{mm})$} & \multicolumn{2}{c|}{ Width(mm) } \\
\cline { 2 - 7 } & Male & Female & Male & Female & Male & Female \\
\cline { 2 - 7 } & 5.58 & 4.72 & 46.13 & 42.82 & 23.30 & 21.79 \\
\hline $\mathrm{P}\left(\mathrm{t}_{0}\right)$ & 0.83 & 0.97 & 2.96 & 2.75 & 1.14 & 1.10 \\
\hline & & $*$ & 0.01 & $*$ & 0.00 & $*$ \\
\hline Forewing & Male & Female & Male & Female & Male & Female \\
\hline AVERAGE & 0.151 & 0.160 & 28.70 & 28.30 & 14.80 & 13.60 \\
\hline STDEV & 0.015 & 0.022 & 1.26 & 1.23 & 0.65 & 0.56 \\
\hline $\mathrm{P}\left(\mathrm{t}_{0}\right)$ & 0.27 & & 0.01 & $*$ & 0.00 & $*$ \\
\hline
\end{tabular}

Table 2. Measurements of the A. dichotoma forewing 


\subsubsection{Forewing strength and optimal forewing structure according to sex}

Table 3 shows the tensile fracture force of the 4-mm-wide male and female specimens, including the thickness of the upper and lower laminations of the forewings and the corresponding stress. The figures above are the mean values obtained for specimens in all orientations as shown in Fig. 15. The T-test results regarding male and female A. dichotoma are also presented in Table 3. Although there is no difference in stress between the male and female forewing, there are significant differences in both the thickness and the tensile fracture force. The forewings of the females are thicker and exhibit a higher tensile fracture force than the males.

\begin{tabular}{|c|c|c|c|c|}
\hline \multirow{2}{*}{ Beetle } & \multirow{2}{*}{ Object } & Force & Thickness & Stress \\
\cline { 3 - 5 } & & $(\mathrm{KN})$ & $(\mu \mathrm{m})$ & $(\mathrm{MPa})$ \\
\hline \multirow{2}{*}{ Male } & Average & 28.1 & 54.0 & 130.7 \\
\cline { 2 - 5 } & STDEV & 5.94 & 7.22 & 25.84 \\
\hline \multirow{2}{*}{ Female } & Average & 35.6 & 69.7 & 127.9 \\
\cline { 2 - 5 } & STDEV & 2.32 & 8.04 & 25.20 \\
\hline \multirow{2}{*}{ T-test } & $\mathrm{P}\left(\mathrm{t}_{0}\right)$ & 0.00 & 0.00 & 0.49 \\
\cline { 2 - 5 } & & $*$ & $*$ & \\
\hline
\end{tabular}

Table 3. Tensile results of the forewing

However, in nature, beetles regularly engage in combat with each other or with insects of other species, either when searching for food or for a mate. For this reason, injured spots can often be found on beetle forewings, which are the result of tearing by the horns of competing beetles. Initially, 20 of the $A$. dichotoma died, most likely due to fighting or other natural causes. Among these beetles, 14 were male and 34 injured spots were identified among the forewings of the 12 wounded beetles, i.e., an average of 2.43 injured spots per male A. dichotoma [Chen et al., 2007a]. Meanwhile, only a single injured spot was located on the forewings of the females. Therefore, it is reasonable to assume that the differences in fighting injuries may be the result of evolutionary adaptations.

The male A. dichotoma has a heavy, large body with a light forewing (see Table 2). The beetle uses its lightweight wing to support its heavy body effectively in flight and uses its horn to protect its body. Observations have indicated that the male has a large but thin forewing with a lightweight construction. The females, who lack horns, have only the forewing to serve as protecting armor. It is thus reasonable to assume that possession of a thick forewing maximizes the female beetle's chance of survival in nature, increasing its lifespan and mating opportunities and further affording it a better opportunity to pass on its genes to the next generation. As a result of the thickened forewing, the beetle's tensile fracture force is increased, which forms a stronger barrier against external damage (see Table 3).

Furthermore, it is also hypothesized that the forewing of the female A. dichotoma is reinforced not only by an increased thickness but also by varying the orientation of its laminated layers and other features. These possible reinforcement mechanisms are areas for future work. The 
following discussion, however, primarily covers the fiber-reinforcement mechanism and the respective design techniques within both the male and female A. dichotoma.

\subsubsection{Optimum cross section of the chitin fibers and their volume fraction in the forewing}

As discussed above, the chitin fibers play a role in fiber enhancement of the forewing. Here, we discuss the "shape" (cross section) and the "amount" (density as a percentage) of reinforced fiber enhancements within the current bio-composite materials.

To discuss the cross sectional shape of the fibers and the distribution of the fiber density in the forewing, the forewing fiber cross sectional information must first be discussed. As shown in Fig. 16(a,b), the forewing consists of an upper lamination, a lower lamination and a void lamination, in which many trabeculae are distributed throughout. The inside of both the upper and lower laminations, called the endocuticle, is composed of chitin fibers (see Fig. 16a, b). The outsides of the lamination regions, called the exocuticle, are composed mainly of proteins. It was observed that the fibers terminated at the exocuticle of the epipleuron tip, and their cross sectional shapes were different from the fibers located at the endocuticle. Cross sections of the fibers that were observed in the exocuticle and the endocuticle are shown in Fig. 17, below. Also in Fig. 17(a, b), the fibers at the epipleuron tip of the forewing can be seen to have a circular cross section with a sparse distribution. Meanwhile, the fibers located primarily around the void lamination in the forewing's endocuticle are densely distributed with a rectangular cross section as seen in Fig. 17(c, d).
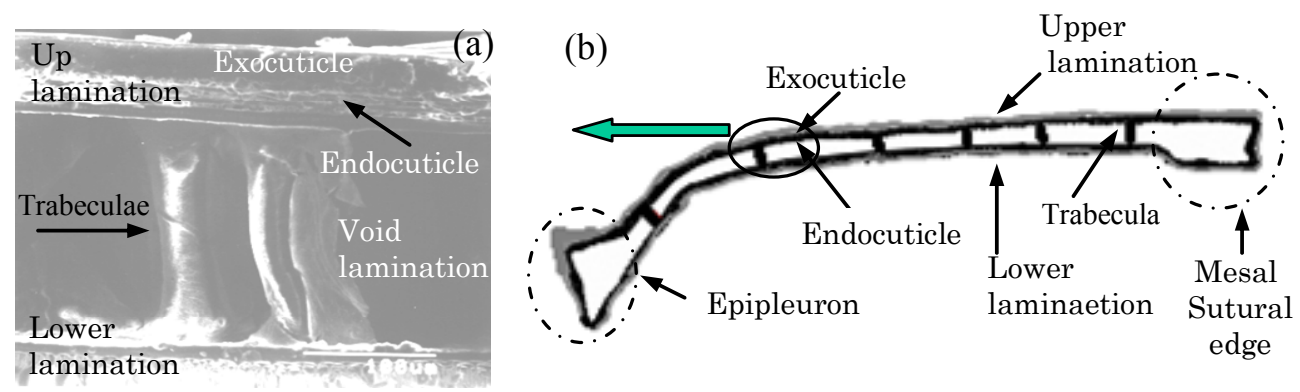

Figure 16. Schematic of section component in the forewing of beetle. (a) micrograph, (b) single model of whole section of forewing.

Fig. 18 illustrates two types of idealized chitin fibers, with either square or circular cross sections. Assuming that both the side of the square and the diameter of the circle are of the same dimension $\mathrm{D}$, then multi-layers of the chitin fibers oriented normally at $90^{\circ}$ in a dense and sparse distribution as shown in Fig. 18 (a) and (b). Under the same densely distributed condition, the chitin fiber volume fraction in the square cross section (Fig. 18a) is approximately $27 \%$ higher than that of the circular (Fig. 18b). It is thus supposed that the fiber reinforcements with rectangular cross sections result in a maximal fiber volume and the high strength of the forewing. 

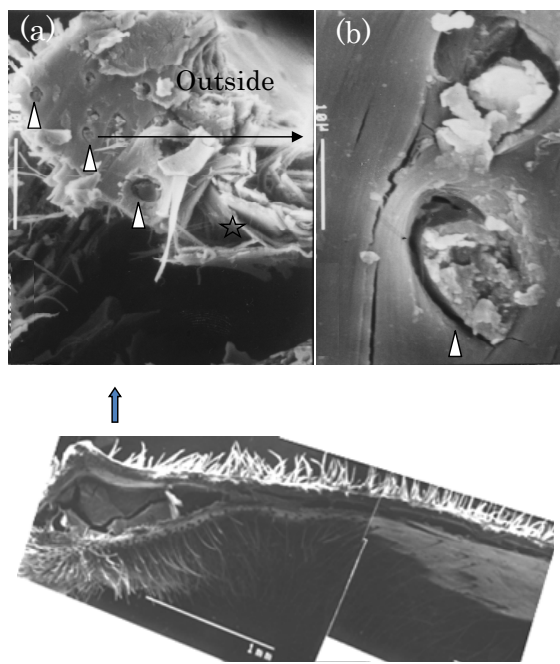

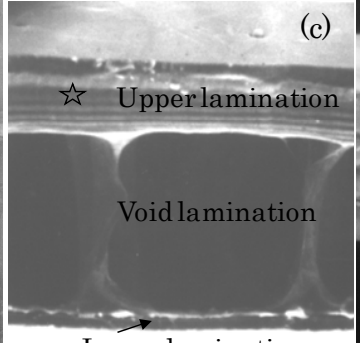

Lower lamination
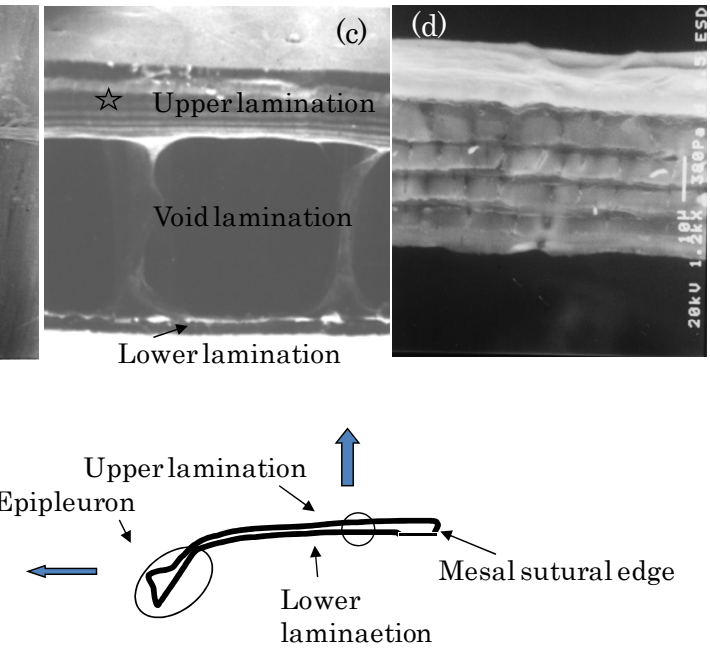

Figure 17. Typical cross sectional shape of the Chitin fibers and their positions in the forewing of $A$. dichotoma, the circular cross section $(\Delta)$ (a) and a magnified view (b) within the epipleuron tip and the rectangular cross section ( $k$ ) (c) around the void lamination in the endocuticle, as well as its magnified view (d).
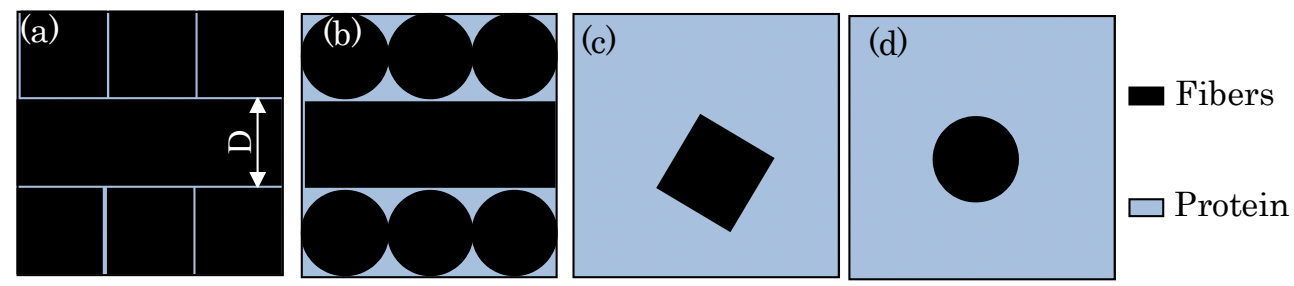

Figure 18. Model of a chitin fiber cross section - Higher density with (a), rectangular and (b) circular shapes and lower density with (c) rectangular and (d) circular shapes.

Next, the sparsely distributed samples of square and circular chitin fibers, as shown in Fig. 18 (c) and (d), have approximately only a 5\% fiber volume fraction. The circular samples have less stress concentration than either rectangular or triangular cross sections would, and they also have a more effective interface with the surrounding protein matrix. Furthermore, the circular samples measure $10 \mu \mathrm{m}$ in diameter and are twice as broad as those of the square cross section in length; its polar moment of inertia is more than ten times higher than that of the rectangular cross sections because it is proportional to the fourth power of the cross section size.

\subsubsection{Optimal structures by kind of beetles}

For comparison, photographs of P. inclinatus (Fig. 19 to Fig. 21) display the sections of the forewing, the trabecular distribution and their structure. Based on these, we confirmed that the fundamental structures of $P$. inclinatus, such as the frame structure (Fig. 19), the chitin 
fiber layers and their arrangement, are similar to that of A. dichotoma. The ratio of the thickness at the edge or the thickness at the central region to the total thickness of both beetles is nearly the same at any position (at the edge or center), but the void thickness in $A$. dichotoma is much larger than that of $P$. inclinatus [Chen et al., 2000].

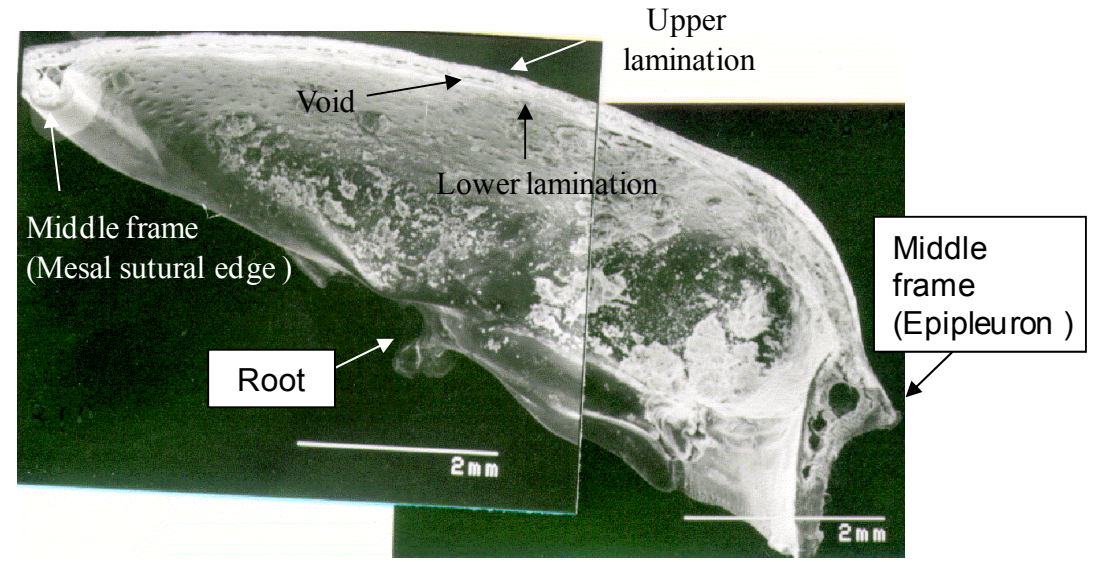

Figure 19. Structure of different sections of the forewing of $P$. inclinatus.

The average density of the trabeculae, calculated as the number of trabeculae per square of $\mathrm{mm}$, is 35 for $P$. inclinatus, and 6 for $A$. dichotoma, which is a difference in trabecular density of approximately six times. The trabeculae can be seen in Fig. 20(a) for A. dichotoma, and Fig. 20(b) for P. inclinatus.
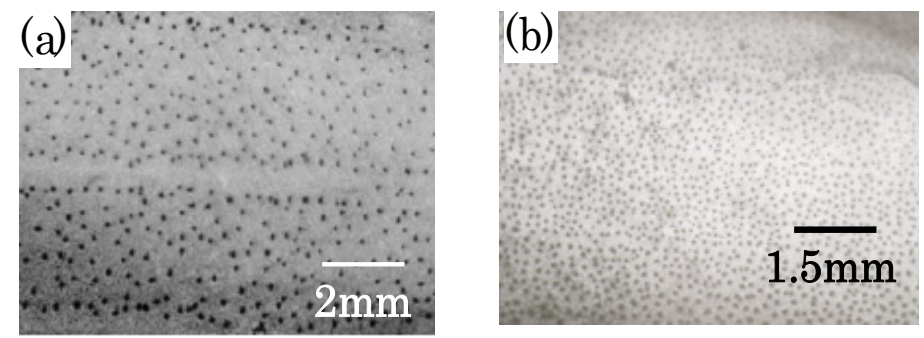

Figure 20. The forewing and trabeculae with $10 \% \mathrm{KOH}$ treatment of (a) A. dichotoma, (b) P. inclinatus.

The trabeculae in A. dichotoma are also relatively long and of a smaller diameter, whereas in $P$. inclinatus, they are relatively short with a larger diameter (Fig. 21a-c).

Here, the differences in structural details between $A$. dichotoma and $P$. inclinatus are discussed. Although both species of beetles possess a lightweight structure based upon a sandwich plate structure and frame construction with variable cross section, there are still measurable differences that exist between the two. For example, P. inclinatus has not only short and large diameter trabeculae but also a trabecular density six times greater than $A$. dichotoma. The forewing of $P$. inclinatus is twice as thick in the upper and lower laminations, 

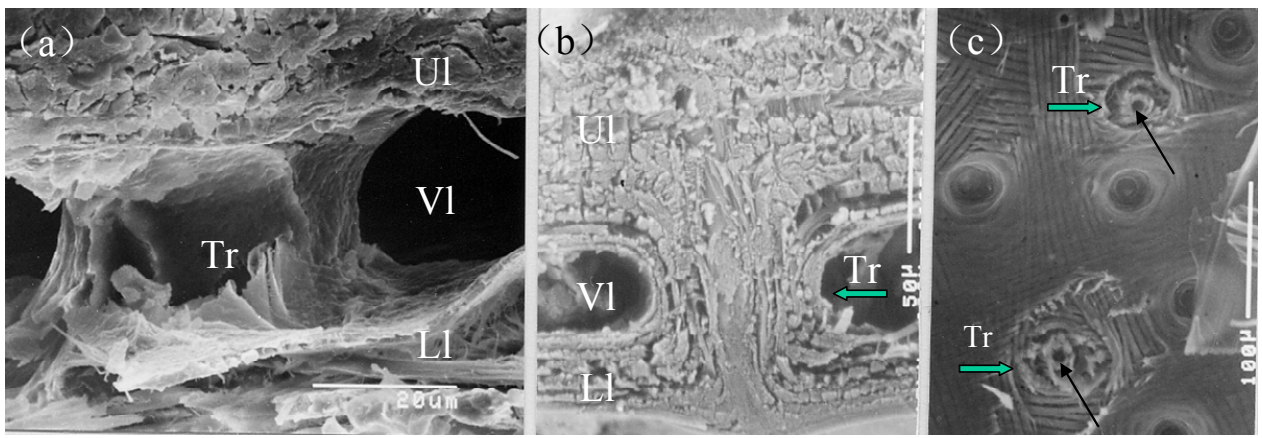

Figure 21. Microstructure of the trabeculae of $P$. inclinatus. (a) whole, (b) in longitudinal section, (c) in upper lamination following treatment with $\mathrm{KOH}$.

and its void height is $40 \%$ that of $A$. dichotoma [Chen et al.,2000]. Based on these differences, we consider that $A$. dichotoma must have a weaker forewing structure, while $P$. inclinatus possesses the higher strength structure. The forewing structure of $P$. inclinatus is optimized mainly by its lightweight structure, which is achieved by varying the quantity parameters, such as trabecular diameter, density, and thickness of lamination.

Why do these two beetles have such obvious structural differences? We have considered that these differences may be related to the lifespan of each beetle species. $P$. inclinatus has a longer lifespan and is able to survive through the winter, but A. dichotoma has a lifespan of only a few weeks and cannot survive the winter. In other words, $P$. inclinatus would be expected to have more durable physical characteristics than $A$. dichotoma because the latter has an extremely short lifespan. From a product viewpoint, the structural design of $P$. inclinatus is meant for durability and longevity, while A. dichotoma is of a more economical design with shorter lifetime. It is also noteworthy that the middle section of the lower lamination of $A$. dichotoma is extremely thin, possessing a thickness of only $1 / 3$ that of $P$. inclinatus. Because this part of the beetle's body is unexposed and thus rarely attacked by enemies in nature, the evolution of an extremely lightweight forewing in A. dichotoma resulted [Chen et al., 2007b].

\subsection{Conclusions}

In this section, the optimal composite structures in the beetle's forewing were discussed based on the beetle's sex, the tensile fracture force of the forewing, the chitin fiber shape and the fiber content. The results are as follows:

1. In comparison to the females, it is clear that the males have lighter and thinner forewings with a lower tensile fracture force; this is because male beetles have a large horn to protect them, while the females do not. The females, with their larger bodies to produce offspring, have only their thick forewings to serve as protecting armor.

2. By investigating the characteristics of the reinforced composite structures within the beetle forewing, it was found that densely distributed chitin fibers were located around 
the void lamination in the endocuticle of the forewing and that they have a rectangular cross section, possessing a maximum fiber volume fraction for reinforcement. Meanwhile, the sparsely distributed cross sections within the exocuticle at the epipleuron tip of the forewing are of a circular cross section and have a strong interfacial reinforcement within a protein matrix.

3. Both beetle species forewings are of the sandwich plate structure with a central void layer, in which there exists many distributed trabeculae. Thus, the beetle forewings are a lightweight composite frame structural design. The structure of the A. dichotoma forewing is disposable and shows an economical design, while the structure of $P$. inclinatus shows a strong and durable design.

\section{Applications of the beetle forewing structure for developing composite materials}

Honeycomb structures are typical lightweight and high-strength composites. There are numerous examples of related studies and applications: from nanomaterials [Kim, 2006; Hideki \& Kenji, 1995] to massive structures, such as those used in F4 fighters, Boeing-767 and Airbus-380 airplanes, and Boeing-360 helicopters [Sato \& Kino, 2004; Shafizadeh et al., 1999; Llorente, 1989]. Presently manufactured commercial honeycomb sandwich plates are produced by adhesively joining the plate parts and core parts, which are made separately by different processes [Leng, 2009; http://www.corecomposites.com; Chen et al., 2008; http://www.nida-core.com/english/contact.htm]. The sandwich plates made by the above processes can be readily separated at the joint of the side plates to the core, and this separation potential is a factor that limits both the strength and the side sealing effectiveness. Furthermore, the use of the adhesive glue is not only environmentally harmful but also expensive [Han et al., 2002; Gu et al., 2010]. To overcome the weaknesses associated with traditional manufacturing method, we have recently developed an integrated molding process [Chen et al., 2012] and the bionic composite sample of the first integrated honeycomb plate is shown in Fig. 22
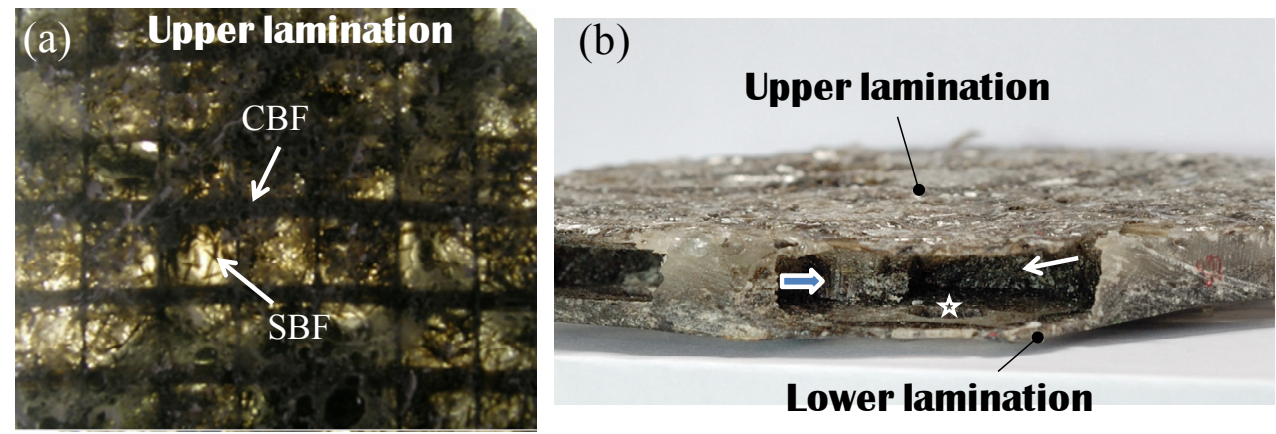

Figure 22. An example of the bionic composite. (a) Front view, (b) side view. CBF denotes continuous basalt fiber; SBF denotes short basalt fiber; the thick and thin arrows indicate the trabeculae and the honeycomb walls, respectively, and the star indicates a processing hole. 
Fig. 23 shows the flow chart of the integrated manufacturing process used to produce the honeycomb plate [Chen et al., 2012]. To improve the association between the BF (Basalt Fiber) and the epoxy resin, the surfaces of the short BFs and CBF (Continuous Basalt Fiber) geogrid were treated with a silane-coupling agent. At a mass ratio of $30 \%$, the BFs were placed in an ethanol solution with $0.75 \% \mathrm{KH} 550$ ( $\gamma$-aminopropyltriethoxysilane); the BFs were removed from the ethanol solution after $30 \mathrm{~min}$ of soaking and were allowed to dry naturally. Finally, the BFs were heat treated for 1 hour at $120^{\circ} \mathrm{C}$ in an oven. At the same time, the mold tools were prepared for the integrated molding, the CBF geogrid was placed in the upper and lower layer, and then the short BFs and epoxy resin were evenly mixed. Table 4 lists the materials used for the mixture and their mass ratios [Chen et al., 2012].

\subsection{Experiment}

Fig. 23 shows the flow chart of the integrated manufacturing process used to produce the honeycomb plate [Chen et al., 2012]. To improve the association between the BF (Basalt Fiber) and the epoxy resin, the surfaces of the short BFs and CBF (Continuous Basalt Fiber) geogrid were treated with a silane-coupling agent. At a mass ratio of $30 \%$, the BFs were placed in an ethanol solution with $0.75 \%$ KH550 ( $\gamma$-aminopropyltriethoxysilane); the BFs were removed from the ethanol solution after $30 \mathrm{~min}$ of soaking and were allowed to dry naturally. Finally, the BFs were heat treated for 1 hour at $120^{\circ} \mathrm{C}$ in an oven. At the same time, the mold tools were prepared for the integrated molding, the CBF geogrid was placed in the upper and lower layer, and then the short BFs and epoxy resin were evenly mixed. Table 4 lists the materials used for the mixture and their mass ratios [Chen et al., 2012].

After pouring the well-mixed fluid of fibers and resin into the mold tools, the mixture was vacuum-dried for 30 minutes at room temperature in a vacuum oven. A portion of aluminum foil and a heavy object were set on the assembly of mold tools after the tools were removed from the vacuum oven. The assembly was then cured for 10 hours at $35{ }^{\circ} \mathrm{C}$ in an incubator. The final molded sample was obtained by removing the paraffin support at $75{ }^{\circ} \mathrm{C}$ in the incubator.

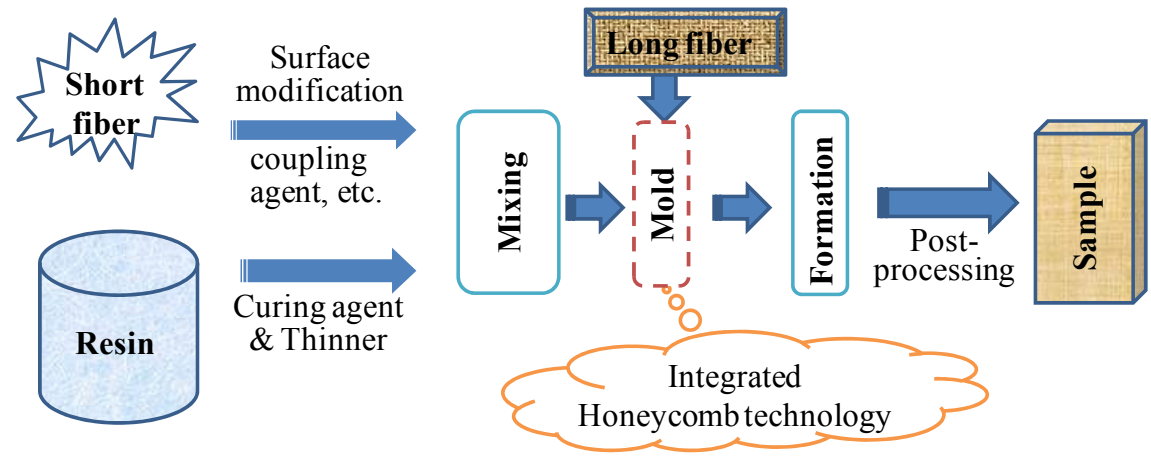

Figure 23. Flow chart of the integrated honeycomb technology. 


\begin{tabular}{|c|c|c|c|c|}
\hline Material & Epoxy resin & Curing agent & Thinner & Short basalt fiber \\
\hline $\mathrm{m} / \mathrm{g}$ & 80 & 20 & 10 & 10 \\
\hline $\mathrm{w} / \%$ & 66.7 & 16.7 & 8.3 & 8.3 \\
\hline
\end{tabular}

Table 4. Main materials and their mass ratios

\subsection{Results and discussion}

In this section, we begin with the examination of the natural edge sealing structure used in the beetle forewing, then we investigate the mechanisms of structure formation and the structure functions, and last, we propose an edge sealing technique for integrated honeycomb processing technology.

\subsubsection{Edge sealing of the forewing integrated honeycomb plates and its biomimetic application}

As mentioned above, the macroscopic structure of the beetle forewing features a sandwich plate construction with extensive internal meshwork (denoted by stars in Fig. 2 a, b) and hundreds of trabeculae (denoted by thick arrows in Fig. 2 a-e). The cross section of the forewing edge frame changes by location (denoted by EFS in Fig. 2a, e). With the exception of the concavo-convex junction between the left (denoted by round arrows, Fig. 2e) and the right forewings, the edge frame is an ingeniously integrated sealing structure without any seams (Fig. 2e). Before the bionic application of the forewing of A. dichotoma could be carried out, a search for the formation mechanisms and an analysis of the structural function were conducted, as discussed in the next section.

\subsubsection{The formation mechanisms of the forewing integrated trabecular honeycomb structure}

To understand the natural mechanisms behind the formation of the forewing integrated honeycomb structure, we first investigated the hexagonally shaped honeycomb structure inside the beetle forewings (Fig. 2a, b); specifically, we were interested in the edge sealing structure without any seams. According to Thompson's cell partitioning theory (Fig. 24) [Thompson, 1945], when a cell membrane intersects with two other cells on a plane, angles $\alpha, \beta$, and $\gamma$ are determined by the reciprocity of the three tensions $t, T$, and $T^{\prime}$ at the intersection O. For example, three scenarios are shown in Fig. 24 (a)-(c) as follows: (a) when $\mathrm{T}^{\prime}=\mathrm{T}>\mathrm{t}$, i.e., when comparing $\mathrm{T}^{\prime}$ and $\mathrm{T}$, $\mathrm{t}$ can be ignored, and in this case, $\alpha$ and $\beta$ are right angles; (b) when $\mathrm{T}^{\prime}=\mathrm{T}>\mathrm{t}$, i.e., $\mathrm{T}^{\prime}$ and $\mathrm{T}$ are quite large compared with $\mathrm{t}$, and thus, $\alpha$ and $\beta$ are obtuse angles; (c) $\alpha, \beta$, and $\gamma$ are all $120^{\circ}$ when $\mathrm{T}^{\prime}=\mathrm{T}=\mathrm{t}$. The mesh structures inside the forewing of the $A$. dichotoma beetle are extensively distributed, and the forewing exhibits a curved structure with thousands of trabeculae and air sacs possessing dissimilar internal stresses. Most of the cells of the forewing have essentially the same function as the branched veins of the dragonfly wing [Thompson, 1945], and the forewing air sacs are distributed in a specific manner to form a $120^{\circ}$ angle between the two adjacent membranes, i.e., they are similar to a honeycomb structure (Fig. 2b). 

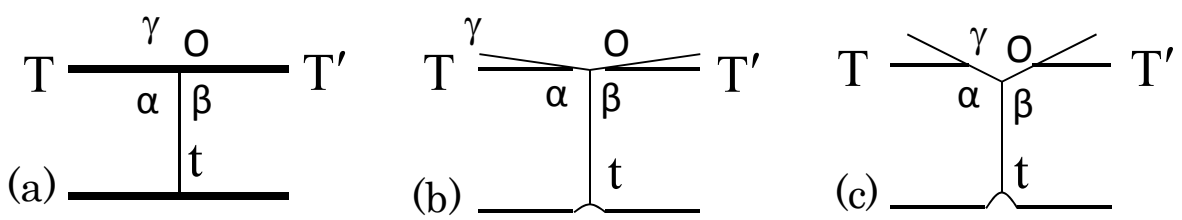

Figure 24. The theoretical cell shape and the relationship between the three tensile forces $\mathrm{T}^{\prime}, \mathrm{T}$ and $\mathrm{t}$ : (a) $\mathrm{T}^{\prime}=\mathrm{T}>\mathrm{t}$, (b) $\mathrm{T}^{\prime}=\mathrm{T}>\mathrm{t}$, (c) $\mathrm{T}^{\prime}=\mathrm{T}=\mathrm{t}$ [Thompson, 1945].

In addition, to better understand why the forewing is constructed with the trabeculae and edge frame of the honeycomb structure, the functions of these structural features, the trabeculae and the edge frame, have been investigated previously [Chen et al., 2000; Chen et al., 2001a; Chen et al., 2007b; Ni et al., 2001]. These investigations have reported the following: the trabeculae and the edge frame provide bending stiffness and resistance to applied stress for the entire forewing structure [Chen et al., 2000; Chen et al., 2007b]. These structures also effectively increase the inter-laminar strength between the lamination layers of the composite structure [Chen et al., 2001a; Ni et al., 2001]. Furthermore, the structures impart the forewing with sufficient mechanical properties to enable the beetle with flight capabilities. These beneficial properties are achieved by the specific angles held between the different fiber layers in the upper lamination of the forewing [Chen et al., 2001a] and the natural design of the transitional region of the edge frame [Chen et al., 2000]. In this manner, the entire forewing of the $A$. dichotoma beetle forms the completely integrated trabecular honeycomb structure.

\subsubsection{Integrated honeycomb plates with edge sealing and the bionic imitation technique}

In the previous section, a complete schematic of a beetle forewing was included to demonstrate the structure of the integrated trabecular honeycomb plate with edge sealing (Fig. 25); in the present work, we did not consider the special functions of the edge frame,
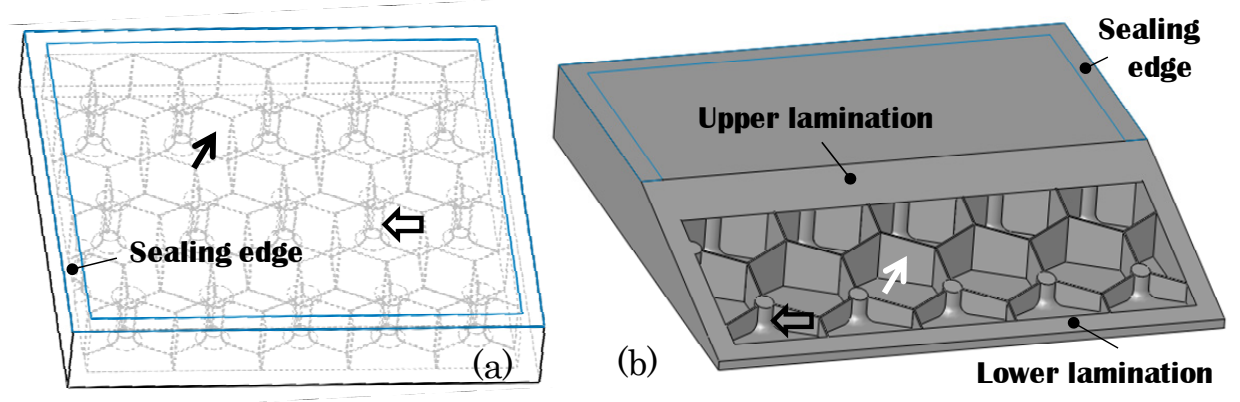

Figure 25. The edge sealing schematic developed for manufacturing the integrated honeycomb, which was inspired by the beetle forewing. (a) Entire integrated honeycomb plate, (b) oblique cross section of (a). The thick arrows indicate trabeculae; the thin arrows indicate honeycomb wall segments. 
and thus, the hollow part of the edge frame was not incorporated. This structure helped us to develop not only a new manufacturing method for producing honeycomb plates but also a new edge sealing technology. Fig. 26a shows a set of mold tools used for sealing operations. By adding extra space around the edge of the female mold [Chen et al., 2012], a sealing edge can be generated. Fig. 26b shows a cross sectional schematic of the sealing edge of the integrated honeycomb plate. This technology does not require bonding or other mechanical connections made by nail or inlay and is therefore preferred over the traditional honeycomb plates because of the simplified manufacturing process, the perfect sealing (as seams are not required) and the enhanced integration.
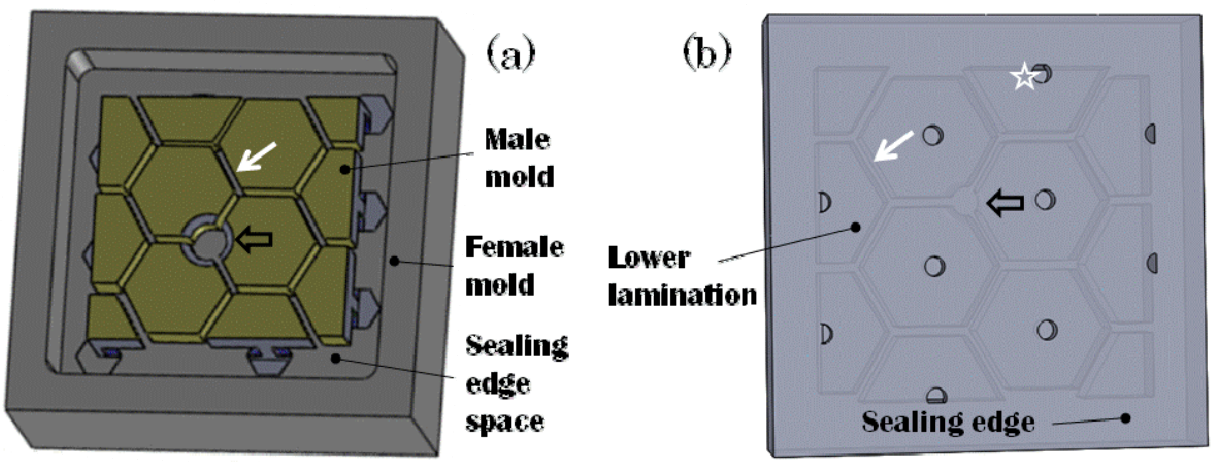

Figure 26. Schematic of the edge sealing approach for use in the integrated honeycomb technology. (a) Mold tools for bionic sealing, (b) image of the integrated honeycomb plate with the sealing edge. The thick arrows indicate trabeculae or the space used to form it; the thin arrows indicate honeycomb wall segments or the space used to form it, and the star indicates a processing hole.

\subsubsection{Processing and optimization of the integrated honeycomb technology}

Optimization of the processing hole In the integrated honeycomb plate, as shown in Fig. 26 , there is a small processing hole in each core. However, the processing holes can have an adverse effect on the strength of the composite and should ultimately be minimized or eliminated. Certain approaches, such as decreasing the diameter of the holes or reducing their number by using integrated male molds (Fig. 27a), may prove effective toward reducing the impact of the processing holes on the composite strength. Increasing the interconnections between the basic male molds to integrate them into the structure can reduce the number of the processing holes needed; however, this requires special molds, as shown in Fig. 27b, c. To accomplish this goal, a thin-walled hexagonal box for each honeycomb unit can be formed and then integrated [Chen et al., 2012]. Integrating the boxes and then fusing them into the plate can help to eliminate the number of processing holes required. 

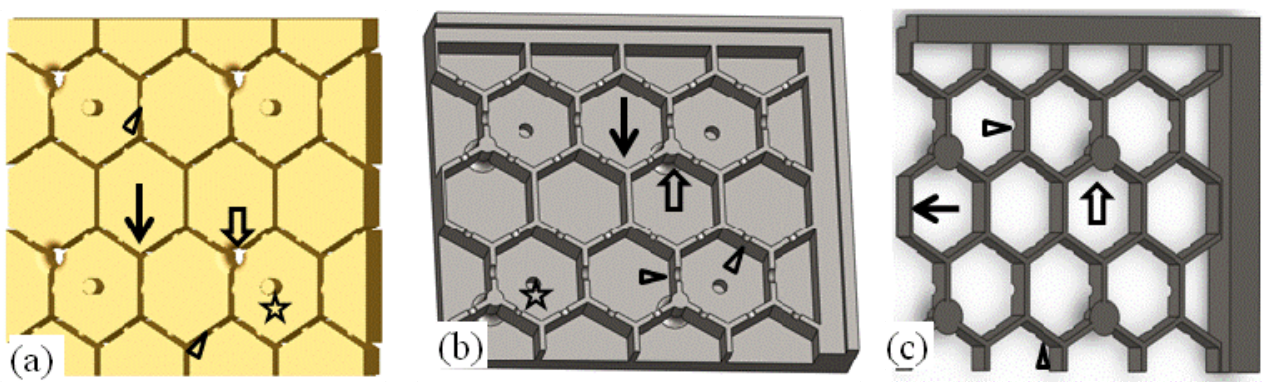

Figure 27. Schematic of the integrated male mold and its mold tools (only a quarter of the symmetric structure is shown in this figure). (a) Bottom view of the integrated male mold, (b) lower half of a mold tool assembly, (c) upper half of a mold tool assembly. The thick arrows show the trabeculae or the space to form them. The thin arrows indicate the honeycomb wall or the space to form them. The star indicates either a positioning hole or a positioning pin. The triangles indicate the processing columns or the space to form them.

Optimization of materials and manufacturing processes As discussed above, the long molding time required for the first method is a drawback. Even so, the molding time can be optimized by selecting appropriate resins or even by using the presently presented process of wax mold tools. For example, two laminating resins, TG-1001 ${ }^{\mathrm{TM}}$ and TG-1009 ${ }^{\mathrm{TM}}$, can be cured in a short period of time (such as $5 \mathrm{~min}$ ) and under a relatively low temperature (33$85^{\circ} \mathrm{C}$ ) [http://www.thermalguard-technology.com]; resin transfer molding and vacuumassisted molding are both performed in this temperature range. Such resins, which are noncombustible, exhibit high strength, are refractory, and meet our complete list of requirements. They also have potential for use in furniture manufacturing, civil engineering, transportation devices, and other applications. The disadvantages of these resins, such as the low content and uneven distribution of fibers, can still be optimized by depositing more fiber-woven roving, by prefilling a certain amount of short fibers into the resin, or by applying vacuum-assisted molding processes.

\subsubsection{Mechanical properties of the integrated trabecular honeycomb structure}

To gain knowledge of the mechanical properties of the integrated trabecular honeycomb structure, we focused on revealing whether fiber interconnections exist between the upper/lower laminations and the trabeculae; these interconnections are considered to be the main difference between the trabecular honeycomb plate and the traditional honeycomb plate. Several studies have previously investigated the mechanical properties of the trabecular honeycomb structure of the beetle forewing as well as bionic materials based on the beetle forewing. First, experiments and analyses using the finite-element method (FEM) were conducted on living forewing specimens and their peeling models; the results indicated that the forewing possesses a high inter-laminar strength value [Ni et al., 2001; Gu et al., 2010]. Second, a bionic composite specimen made from a sandwich structure with fiber-reinforced trabeculae was constructed; the experimental results demonstrated that the 
energy release rate and the maximum shear stress of the fiber-reinforced material were approximately four times greater than those of a traditional plate without fiber-reinforced trabeculae [Okazaki et al., 2005]. Third, the energy-absorbing capabilities of the original and improved models of the beetle forewing were analyzed using nonlinear FEM, and it is clear from the models that the forewing structure is both crashworthy and energy-absorbing, particularly in the improved models [Guo \& Wang, 2011]. Furthermore, the trabecular honeycomb plates that were produced by our bionic method not only achieved integrated preparation between the upper and lower lamination and the core lamination but also produced a natural seamless edge using the new sealing technology. Additionally, the reinforcing fibers were distributed between the core layer (trabeculae, honeycomb walls) and the upper and lower laminations (including the edge part); that is, the bionic materials of the real integration trabecular honeycomb plate are similar to the biological structure of the beetle forewing. Therefore, the bionic plate should exhibit excellent mechanical properties, as suggested by the aforementioned physical experiments and FEM analysis.

\subsection{Conclusions}

The processes for manufacturing integrated honeycomb plates have been outlined, and the problems associated with the integrated honeycomb technology have been discussed and resolved:

1. Each forewing of a beetle has a natural edge sealing design with a completely integrated structure consisting of honeycomb cores, including trabeculae and an edge frame. The formation mechanism of the integrated trabecular honeycomb structure and its mechanical properties were discussed. An edge sealing technique inspired by the forewing structure was proposed. This technique has numerous advantages, such as processing simplicity and complete seamless integrated.

2. For the first time, detailed manufacturing processes have been developed and presented toward achieving integrated honeycomb plates that include edge sealing. Optimization methods have been developed, such as integrating the male molds that can eliminate the influence of the processing holes and choosing a fast-curing and hightemperature resin that can shorten the molding time.

\section{Author details}

Jinxiang Chen* and Juan Xie

International Institute for Urban Systems Engineering \& School of Civil Engineering, Southeast

University, China

Qing-Qing Ni

Dept. of Functional Machinery \& Mechanics, Shinshu University, Japan

\footnotetext{
${ }^{*}$ Corresponding Author
} 


\section{Acknowledgement}

This work was supported by the Natural Science Foundation of China (Grant No. 51173026), the Jiangsu NSF (No.BK2010015) and the National Key Technologies R\&D Program of China (2011BAB03B10).

\section{References}

Banerjee, S. (1988) Organisation of Wing Cuticle in Locusta Migratoria Linnaeus, Tropidacris Cristata Linnaeus and Romalea Microptera Beauvais (Orthoptera: Acrididae). International Journal of Insect Morphology and Embryology, Vol. 17, pp 313-326.

Ben, G.; Nishi, Y. \& Asano, S. (1998) A Discussion of Mechanical Propreties for an Optimum Stackling Sequence in Beetles. Nippon Kikai Gakkai Zairyo Rikigaku Bumon Koenkai Koen Ronbunshu, Vol. 1998-B, pp 349-350.

Chen, J.; Dai, G.; Xu, Y. \& Iwamoto, M. (2007a) Optimal Composite Structures in the Forewings of Beetles. Journal of Composite Structures, Vol. 81, pp 432-437.

Chen, J.; Gu, C.; Guo, S.; Wan, C.; Wang, X.; Xie, J. \& Hu, X. (2012) Integrated Honeycomb Technology Motivated by the Structure of Beetle Forewings. Journal of Bionic Engineering. doi:10.1016/j.msec.2012.04.067.

Chen, J.; Iwamoto, M.; Ni, Q.Q.; Kurashiki, K. \& Saito, K. (2000) Cross Sectional Structure and its Optimality of the Forewing of Beetles. Journal of the Society of Materials Science, Vol. 49, No. 4, pp 407-412.

Chen, J.; Iwamoto, M.; Ni, Q.Q.; Kurashiki, K. \& Saito, K. (2001a) Laminated Structure and its Mechanical Properties of the Forewing of Beetle. Journal of the Society of Materials Science, Vol. 50, pp 455-460.

Chen, J.; Ni, Q.Q.; Endo, Y. \& Iwamoto, M. (2001b) Fine Structure of the Trabeculae in the Forewing of Allomyrina Dichotoma (Linne) and Prosopocoilus Inclinatus, (Motschulskey) (Coleoptera: Scarabaeidae). Insect Science, Vol. 8, pp 115-123.

Chen, J.; Ni, Q.Q; Endo, Y. \& Iwamoto, M. (2002) Distribution of the Trabeculae in the Forewing of Horned Beetle, Allomyrina Dichotoma (Linne) (Coleoptera: Scarabaeidae). Insect Science, Vol. 9, pp 55-61.

Chen, J.; Ni, Q.Q.; Li, Q. \& Xu, Y. (2005) Biomimetic Lightweight Composite Structure with Honeycomb-trabecula. Acta Materiae Compositae Sinica, Vol. 22, pp 103-108.

Chen, J.; Ni, Q.Q.; Xu, Y. \& Iwamoto, M. (2007b) Lightweight Composite Structures in the Forewings of Beetles. Journal of Composite Structures, Vol. 79, pp 331-337.

Chen, J.; Xie, J.; Zhu, H.; Guan, S.; Wu, G.; Noori, M.N. \& Guo, S. (2012) Integrated Honeycomb Structure of a Beetle Forewing and its Imitation. Materials Science $\mathcal{E}$ Engineering C, Vol. 32, pp 613-618.

Chen, X.; Sun, Y. \& Gong, X. (2008) Honeycomb Textile Composites, and Experimental Analysis of 3D Honeycomb Textile Composites Part I: Design and Manufacture. Textile Research Journal, Vol. 78, No. 9, pp 771-781. 
Gu, R.; Guo, K.; Qi, C.; Zhang, J. \& Liu, Z. (2010) Properties and Bonding Mechanism of Konjak Powder-chitosan-PVA Blending Adhesive. Transactions of the CSAE, Vol. 26, No. 5, pp 373-378.

Gullan, P.J. \& Cranstion, P. (1994) The Insects: An Outline of Entomology. Chapman \& Hall, pp 22-55.

Guo, T.; Wang, Y.F.; (2011) Energy Absorbing Structures Imitating Trabecular of Beetle Cuticles. Engineering Mechanics, Vol. 28, No. 2, pp 246-251.

Han, T.S.; Ural, A.; Chen, C.S.; Zehnder, A.T.; Ingraffea, A.R.; Billington, S.L. (2002) Delamination Buckling and Propagation Analysis of Honeycomb Panels Using a Cohesive Element Approach. International Journal of Fracture, Vol. 115, pp 101-123.

He, M. \& Cox, B.N. (1998) Crack Fridging by Through-Thickness Reinforcement in Delaminating Curved Structures, Composites Part A, Vol. 29A, pp 377-393.

Hepburn, H.R. (1976) The Insect Integument. Elsevier scientific Publication Company,New York, pp 1-100.

Hideki, M. \& Kenji, F. (1995) Ordered Metal Nanohole Arrays Made by a Two-Step Replication of Honeycomb Structures of Anodic Alumina. Science, Vol. 268, No. 5216, pp 1466-1468.

Hosur, M.V.; Abdullah, M.; Jeelani, S. (2005) Manufacturing and low-velocity impact characterization of foam filled 3-D integrated core sandwich composites with hybrid face sheets. Composite Structures, Vol. 69, No. 2, pp 167-181.

http://www.corecomposites.com

http://www.thermalguard-technology.com.

http://www.nida-core.com/english/contact.htm

Ishii, S. (1982) Physiology of Insects. Baifukan press, pp 40-57.

Iwamoto, M.; Chen, J.; Ni, Q.Q.; Kurashiki, K. \& Saito, K. (1999b) Structure Optimality with

Biomimetics of the Fore-wing of Beetles. The 3rd China-Japan Joint Conference on Composites, HangZhou, pp 15-20.

Iwamoto, M.; Ni, Q.Q.; Fujwara, T. \& Kurashiki, K. (1999a) Interlaminar Fracture Mechanism in Unidirectional CFRP Composites-Part I: Interlaminar Toughness and AE Characteristics. Engineering Fracture Mechanics, Vol. 64, pp 721-745.

Jung, W.Y. \& Aref, A.J. (2005) Analytical and Numerical Studies of Polymer Matrix Composite Sandwich Infill Panels. Composite Structures, Vol. 68, pp 359-370.

Kim, H.; Kim, J.; Yang, H.; Suh, J.; Kim, T.; Han, B.; Kim, S.; Kim, D.S.; Pikhitsa, P.V. \& Choi, M.; (2006) Parallel Patterning of Nanoparticles via Electrodynamic Focusing of Charged Aerosols. Nature Nanotechnology, Vol. 1, pp 117-121.

Leopoldj, R.A.; Newman, S.M. \& Helgeson, G. (1992) A Comparison of Cuticle Deposition during the Pre-and Post-eclosion Stages of the Adult Weevil, Anthonomus Grandis Boheman. International Journal of Insect Morphology and Embryoogyl, Vol. 21, pp 37-62. 
Llorente, S. (1989) Honeycomb Sandwich Primary Structure Applications on the Boeing Model 360 Helicopter. Society for the Advancement of Material and Process Engineering, Vol. 34, No. 5, pp 824-838.

Mania, R. (2005) Buckling Analysis of Trapezoidal Composite Sandwich Plate Subjected to In-plane Compression. Composite Structures, Vol. 69, pp 482-490.

Masuda, Y.; Okada, A.; Tabata, H.; Yoneda, K. \& Yamamoto, Y. (1995) Structure and mechanical properties of the elytra of pachyrhynchid weevils. Lecture Article of L,ecture Meeting of Japan Mechanical Society Department of Materials Mechanics, pp 121-122.

Matsunaga, H. (2002) Assessment of a Global Higher-order Deformation Theory for Laminated Composite and Sandwich Plates. Composite Structures, Vol. 56, pp 279291.

Nguyen, V.T.; Caron, J.F. \& Sab, K. (2005) A Model for Thick Laminates and Sandwich Plates. Composites Science and Technology, Vol. 65, pp 475-489.

Ni, Q.Q.; Chen, J.; Iwamoto, M.; Kurashiki, K. \& Saito, K. (2001) Interlaminar Reinforcement Mechanism in a Beetle Fore-Wing. JSME International Journal Series C, Vol. 44, No. 4, pp 1111-1116.

Okazaki, J.; Ni, Q.Q; Iwamoto, M. \& Kurashiki, K. (2005) Research on Design of a Composite Material Imitated from the Fore-wing of a Beetle. Third International Workshop on Green Composites, pp 91-94.

Sato, T. \& Kino, Z. (2004) Exploration for application business of honeycomb cores and composite materials. Function and Materials, Vol. 24, pp 64-73.

Sekine, H. \& Kamiya, S. (1987) Analysis of a Shear Fracture in Unidirectional FiberReinforced Composites II (For the Case That the Displacement on Boundaries is Constrained in the Direction Normal to the Shear Fracture). Japan Society Mechanical Engineers, Vol. 53, No. 489A, pp 930-934.

Shafizadeh, J.E; Seferis, J.C.; Chesmar, E.F. \& Geyer, R. (1999) Evaluation of the in-service performance behavior of honeycomb composite sandwich structures. Journal of Materials Engineering and Performance, Vol. 8, No. 6, pp 661-668.

Tanimoto, T.; Chen, Q.H. \& Taguchi, M. (1998) Damage Tolerance of biomimetic CFRP Laminate. Abstacts of 51th Annual Meeting of Textile Mechanical Society, pp 78-79.

Thompson, D. W. (1945) On Growth and Form. Cambridge University press, pp 1-131.

Turss, R.W.; Hine, P.J. \& Duckett, R.A. (1997) Interlaminar and Intralaminar Fracture Toughness of UniaxialContinuous and Discontinuous Carbon Fiber/Epocy Composites. Composites Part A: Applied Science and Manufacturing, Vol. 28, pp 627-636.

Leng, L. (2009) An Edge-sealed Table Top Board with Paper Honeycomb Core. Patent (Uk) No. 0722071.8.

Wainwright, S.A.; Biggs, W.D.; Currey, J.D. \& Gosline, J.M. (1976) Mechanical Design in Organisms. Princeton University Press, pp 158-170.

Yoshikawa, H. (1985) The Statistical Analysis Procedure by the Portable Calculator. Japan Union Science Engineering Press publisher, pp 28-32. 
Zelazny, B. \& Neville, A.C. (1972) Quantitative Studies on Fibril Orientation in Beetle Endocuticle. Insect Physiology, Vol. 18, pp 2095-2121. 